ARTÍCULOS 



\title{
VARIACIONES EN LA DENSIDAD DEL OXÍGENO EN EL AIRE Y SU INFLUENCIA SOBRE LA SALUD HUMANA
}

\author{
Jorge Olcina Cantos y David Martín Estévez \\ Universidad de Alicante
}

\section{RESUMEN}

La falta de oxígeno puede conllevar serias consecuencias en la salud de los seres vivos, incluida la muerte. La modificación de su densidad en el aire pueda tener efectos sobre la salud humana, especialmente en grupos de riesgo y personas con afecciones pulmonares o cardiacas previas. Se analiza el efecto de las variaciones en la densidad de oxígeno, originadas por los cambios en las masas de aire presentes en la atmósfera, sobre los ingresos hospitalarios por enfermedad cardiaca y vásculo-cerebral, registrados en la ciudad de Alicante. Se valoran los posibles efectos del cambio climático en el aumento del riesgo frente a enfermedades cardiacas y pulmonares.

Palabras clave: Densidad de oxígeno en el aire, situación atmosférica, sistemas de alerta temprana, efectos del cambio climático.

\section{ABSTRACT}

The absence of oxygen can lead to serious health consequences of living organisms, including death. Any change in its density in the air can have consequences for human health, especially in risk groups, and people with heart or pulmonary affections previous. This paper analyzes the effect of variations in the density of oxygen, caused by changes in air masses in the atmosphere, on hospital admissions for cardiac and cerebrovascular diseases, registered in the city of Alicante. In this paper we study the effects of climate change on the increase in risk from cardiac and pulmonary diseases.

Fecha de recepción: mayo 2010.

Fecha de aceptación: febrero 2012. 
Key words: Density of oxygen in the air, weather situation, early warning systems, climate change.

\section{INTRODUCCIÓN: CLIMA Y SALUD, UNA ESTRECHA RELACIÓN}

La importancia del clima como fuente salutífera ha sido destacada por diversos autores desde la Antigüedad. No faltan ejemplos de la estrecha relación entre clima, temperie y salud; es evidente el malestar físico que causan ciertos tipos de tiempo, como olas de frío o de calor, en personas de salud débil, no acostumbradas a los rigores térmicos de aquéllos, o el disconfort que crean los climas de influencia marítima a personas con afecciones asmáticas. Son numerosos los ejemplos del carácter salutífero o malsano de algunos vientos. Glacken (1996) señaló como los griegos, dentro de las teorías del medio, concedieron al clima y, más en concreto a las temperaturas, el carácter distintivo de su civilización. En este contexto las teorías del medio basadas en la físiología se desarrollaron a partir de la noción de salud y enfermedad como equilibrio o falta de equilibrio humoral (teoría de los humores) y de observaciones como las ventajas de ciertas ciudades o moradas, la situación en relación con la altitud, la proximidad del agua o ciertos vientos dominantes.

La relación entre clima y salud que se plasma ya en la obra del sabio griego Hipócrates se basada en la idea de la estimulación mental y física que provoca la vivencia de un clima saludable o vigorizante. En las teorías del medio que se fraguan en época griega el clima es origen de la distribución espacial de los pueblos, de las diferencias raciales, de las costumbres e incluso de las formas políticas de gobierno. Estos aspectos, con ciertas matizaciones, se mantendrán en el pensamiento occidental hasta los primeros decenios del siglo XIX cuando pierdan razón de ser los postulados del determinismo ambiental.

Superado este paradigma determinista que relacionaba las enfermedades infecciosas con los aspectos del tiempo atmosférico, merced a los avances médicos y al descubrimiento de los microbios y de medicamentos que los combatían, la atención en el estudio de las relaciones entre la salud y las condiciones ambientales se centrará, a lo largo del siglo XX, en las enfermedades crónicas, llamadas enfermedades de la modernidad, esto es, aquellas patologías vinculadas con los sistemas circulatorio y respiratorio.

En efecto, los trabajos de relación entre clima y salud encuentran, desde las primeras décadas de la centuria pasada, continuidad en algunas líneas de investigación concretas. Por un lado, aquellas aportaciones orientadas a determinar la irrupción y propagación de enfermedades en relación con las situaciones meteorológicas. Esta línea de estudio encuentra sus antecedentes en los trabajos sobre la relación entre el sufrimiento causado por ciertos dolores corporales y el desarrollo de ciertas situaciones atmosféricas llevados a cabo por el doctor S. Weir Mitchell, quién vinculó el dolor padecido por algunos pacientes durante el conflicto civil americano a condiciones meteorológicas de baja presión, ascenso de temperatura y humedad y lluvias registradas algunas jornadas; esta hipótesis sería confirmada, durante la Segunda Guerra Mundial, por los estudios de Otto Hoflich sobre la relación entre el dolor y el paso de frentes cálidos en superficie. Por otra parte, las sensaciones experimentadas por el cuerpo humano en virtud de las relaciones existentes entre diversos elementos climáticos, 
será otra de las líneas de investigación sobre el clima y salud desarrolladas en el siglo XX y que culmina con la formulación de índices de sensación térmica o de confort climático. En este sentido destacan los trabajos de R.G Steadman y sus propuestas de tablas de temperatura efectiva. Por último, deben citarse las aproximaciones desarrolladas en el contexto del desarrollo sostenible y la búsqueda de calidad ambiental en medios urbanos como fórmula para la mejora de la salud humana.

En este contexto se inscribe este trabajo. Se analiza la relación entre la variabilidad de oxígeno presente en el aire y el impacto que tiene esa variación en personas con patologías relacionadas con los aparatos circulatorio y respiratorio de un territorio concreto. Para ello, es fundamental el estudio de las condiciones atmosféricas generales en determinados períodos del año sobre un entorno geográfico concreto.

\section{DENSIDAD DE OXÍGENO EN EL AIRE COMO FACTOR DE RIESGO EN ENFERMEDADES CARDIACAS, VÁSCULO-CEREBRALES Y PULMONARES}

La falta de oxígeno puede conllevar serias consecuencias en la salud de los seres vivos, incluida la muerte. El oxígeno, uno de los componentes principales de la masa atmosférica que nos envuelve, es elemento básico para la vida y, de ahí, que cualquier modificación en su densidad en el aire pueda tener consecuencias sobre nuestra salud - hipoxia-, especialmente, en personas con afecciones pulmonares o cardiacas.

El porcentaje de oxígeno en el aire no varía sustancialmente en la troposfera, manteniéndose en torno al 21\%; esto es, existe la misma proporción a nivel del mar y a siete mil metros de altitud. Otra cuestión es la densidad de la masa atmosférica, que es inversamente proporcional a la altitud; de manera que a siete mil metros de altitud, la masa atmosférica es más liviana que a nivel del mar. Se suele decir, erróneamente, que a gran altitud hay «menos oxígeno» cuando lo que ocurre es que se reduce la densidad del mismo en el aire. De ahí que el peso de la presión atmosférica que soporta nuestro cuerpo sea menor a mayor altitud. Esta disminución del peso de la presión atmosférica no se debe, pues, a un descenso en el porcentaje de oxígeno en aire, sino a la disminución en el número de moléculas de este gas, igual que sucede con otros gases que componen la masa atmosférica. Como ejemplo, a unos cinco mil metros de altitud, la disponibilidad de oxígeno resulta la mitad de la que existe a nivel de mar.

Con estos parámetros físicos fundamentados en la Ley de los gases ideales y, derivada de ellos, en la ecuación de estado y teniendo presente, además, que la presión atmosférica puede ser considerada como la suma de las presiones parciales de los componentes que integran el aire (el hidrógeno, el oxígeno, el vapor de agua, etc.), la doctora Ovcharova estableció, en 1958, una asociación experimental entre la actividad nerviosa superior en animales de laboratorio y el intercambio gaseoso de éstos con el ambiente circundante ${ }^{1}$. Por esos años, los

1 Ovcharova, V.F. (1958): Changes in the superior nervous activity and the gas exchange during the adaptation process of laboratory animals exposed to different weather conditions [in Russian]. Original Lecture. Library of the Instute of Geography, Academy of Sciences of the former USSR, Moscow [unpublished]. Citado por Lecha (1987). 
científicos Voronin $(1954)^{2}$, Chubukov $(1956)^{3}$ habían publicado estudios sobre las relaciones entre actividad nerviosa y cambios en condiciones ambientales. En un trabajo conjunto Voronin, Ovcharova y Spiridonov (1963) afirmarían que: «el organismo humano responde a los cambios inusuales en el tiempo atmosférico y a las variaciones estacionales. La respuesta del cuerpo humano a estos cambios se puede ver sobre todo a través del aumento de la actividad nerviosa, cambios bruscos del sistema de termorregulación y el balance de calor del cuerpo, así como en la actividad cardiovascular».

En 1963, Ovcharova comprobó que la variación en la densidad de oxígeno en el aire producía afecciones directas en la actividad nerviosa superior de estos animales, señalando que ello podría ocurrir igualmente en los seres humanos ${ }^{4}$.

Años más tarde, Ovcharova estableció su índice de densidad de oxígeno en el aire como indicador complejo de los efectos de los cambios del tiempo en la salud humana (Ovcharova, $1981)^{5}$.

El índice complejo de densidad parcial de oxígeno en el aire (DOA) medido en $\mathrm{g} / \mathrm{m}^{3}$, para calcular las diferencias de volumen de oxígeno en aire es el siguiente:

$$
\mathrm{DOA}=80,51 \cdot \mathrm{P} /(\mathrm{T}+\mathbf{2 7 3}) \cdot(1-\mathrm{e} / \mathrm{P})
$$

T: es la temperatura del aire, en grados centígrados.

e: es la tensión parcial de vapor del agua, en hPa.

P: es la presión atmosférica, en $\mathrm{hPa}$.

En el decenio de los años ochenta del pasado siglo, las ideas de la doctora Ovcharova fueron recuperadas por diversos investigadores cubanos que desarrollaron estudios sobre las relaciones entre el tiempo atmosférico y la salud de los seres humanos. Lecha Estela ${ }^{6}$ y su equipo de investigación comenzaron a aplicar el indicador de densidad de oxígeno en aire, buscando el establecimiento de pautas de comportamiento entre la modificación de la densidad en volumen de oxígeno en aire y determinadas patologías de tipo cardiovascular y de tipo respiratorio en latitudes tropicales ${ }^{7}$.

La Internacional Society of Biometeorology (ISB), a través del grupo de trabajo Climate and Human Health, comenzó a desarrollar en los años noventa del pasado siglo vías

2 Voronin, I.M. (1954): Experimental study of the effects of climatotherapy in human organism [in Russian]. In: Proceeding of the 2nd Interdisciplinary Conference on Applications of Climatotherapy. Moscow, November; 25:27. Citado por Lecha (1987).

3 Chubukov, L.A. (1956): Climate fundaments of climatotherapy [in Russian]. In: Basis of Climatotherapy, Vol, 1, Medical Ed., Moscow; 87 pp. Citado por Lecha (1987).

4 Ovcharova, V.F. (1963): Changes in the superior nervous activity and the gas exchange during the adaptation process of laboratory animals exposed to seasonal climate variations [in Russian]. In: Problems of Complex Climatology, USSR Academy of Sciences Ed., Moscow; 141-149. Citado por Lecha (1987).

5 Ovcharova, V.F. (1981): Calculation of the oxygen content of the air based upon meteorological parameters (pressure, temperature and humidity) to forecast the effects of hypoxia sensations [in Russian]. Jour. Questions of Climatotherapy, Physiotherapy and Physical Culture, Vol. 2; 29-34. Citado por Lecha (1987).

6 Lecha, L. (1987): Las sensaciones de calor sofocante en la región central de Cuba. Sciences of the Space and Earth Journal, 13; 56-58.

7 Lecha L. and Delgado T. (1996): On a regional health watch \& warning system. In Proceedings of the 14th Int. Congress of Biometeorology, Ljubljana, Slovenia; Part. 2, Vol. 3; 94-107. 
de trabajo para constatar los impactos en diferentes entornos globales con la aplicación de la metodología comentada y derivada de los estudios de Ovcharova. Surgen así grupos de investigación, en diferentes países, que trabajan la relación entre densidad de oxígeno y su impacto en enfermedades cardiovasculares $(E C V)^{8}$ y pulmonares. En España, Fernandez de Arróyabe analizó, con datos de ingresos diarios del hospital de Basurto (Bilbao), los impactos de la variación del volumen de oxígeno en aire en diferentes patologías a través de estadísticas en esa ciudad de ingresos hospitalarios ${ }^{9}$. En esta misma línea de trabajo se encuadran estudios realizados para la ciudad de Alicante y que tienen por objeto comprobar el comportamiento de la variación en volumen de oxígeno en aire en un ámbito mediterráneo ${ }^{10}$.

Este tipo de aproximaciones se está llevando a cabo, asimismo, por equipos de trabajo en la ciudad de Sao Paulo (Brasil) y en Portugal, donde se analiza también la presencia de ozono ambiental y los impactos en los sistemas respiratorio y cardiovascular ${ }^{11}$.

En la actualidad, se mejoran los métodos de trabajo en estas investigaciones incorporando el efecto de la vorticidad de los campos de presión y añadiendo nuevos parámetros para el diseño de índices biometeorológicos.

Del análisis de la relación entre la variación en la densidad parcial de oxígeno en el aire y su impacto en personas incluidas en grupos de riesgo, es decir, con patologías previas o con factores de riesgo capaces de combinarse y producir el impacto, se han obtenido resultados destacados en diferentes ámbitos regionales. Así, en el ámbito tropical se han obtenido evidencias claras de la relación entre episodios pronosticados de hipoxia e hiperoxia y desarrollo de diferentes enfermedades (Lecha et. alt., 2007). La patología que más se ve afectada por condiciones atmosféricas de hipoxia e hiperoxia es el asma bronquial y, en menor porcentaje, las enfermedades el corazón (a partir de ahora denominadas como EC). Los cambios atmosféricos vinculados con falta o exceso de oxígeno en el aire producen respuestas meteorotrópicas en la población local. Lecha Estela ha identificado, asimismo, una preferencia de los máximos diarios de algunas enfermedades en relación con tipos sinopticos concretos; asi, por ejemplo, las crisis masivas asociadas a la hipertensión arterial, las cefaleas y los accidentes vasculo cerebrales $(\mathrm{AVC})^{12}$ muestran preferencia en condiciones de hipoxia.

8 ECV: Enfermedades cardiovasculares. Según la Organización Mundial de la Salud, incluyen las siguientes patologías: cardiopatía coronaria, enfermedades cerebrovasculares, arteriopatías periféricas, cardiopatía reumática, cardiopatías congénitas, trombosis venosas profundas y embolias pulmonares. Por otro lado, también la OMS apunta que los ataques al corazón y los accidentes vasculares cerebrales (AVC) — en la actualidad denominados enfermedad vasculo cerebral (EVC) - suelen ser fenómenos agudos que se deben sobre todo a obstrucciones que impiden que la sangre fluya hacia el corazón o el cerebro. La causa más frecuente es la formación de depósitos de grasa en las paredes de los vasos sanguíneos que irrigan el corazón o el cerebro. Las EVC también pueden deberse a hemorragias de los vasos cerebrales o coágulos de sangre.

9 Fernández de Arróyabe, H. P.; Lecha, E. L. (2008) «Validación en el norte de España de dos sistemas de alerta sanitar ios basados en la idea del contraste meteorológico extremo», En Cambio climático regional y sus impactos. VI Congreso Internacional AEC. Serie A, ponencia V. Tarragona 2008.

10 Martín Estévez, David (2010): «Estudio para la mitigación de los impactos del tiempo sobre la salud humana: Una aproximación desde la climatología aplicada». Proyecto de investigación para la consecución del Diploma de Estudios Avanzados (DEA). Instituto Interuniversitario de Geografía. Universidad de Alicante.

11 Azevedo, M. J.; Gonçalves, L.T.F.; Andrade, M.F. (2011): «Long-range ozone transport and its impact on respiratory and cardiovascular health in the north of Portugal». International Journal of Biometeorology, 55:187202.

12 AVC: Accidente Vasculo Cerebral. Todos los relacionados con el sistema vascular y que afectan al cerebro. Actualmente se le denomina Enfermedad Vasculo Cerebral (EVC), diferenciando de accidente, ya que los proble- 
En España, como se ha señalado, Fernández de Arróyabe ha trabajado en la variación de la densidad de oxígeno en aire y su impacto en determinadas patologías, a partir del análisis de datos de asistencias hospitalarias (Fernández de Arróyabe y Lecha Estela, 2008). En una situación de hipoxia extrema (varios días con descenso del contenido de oxígeno en la atmósfera) se detectó siempre una respuesta masiva por encima del percentil 66 en las atenciones por accidentes vasculo cerebrales, coincidiendo con los resultados obtenidos por Lecha Estela en latitudes tropicales. Por el contrario, las patologías del corazón, no mostraron un patrón tan claro y su ocurrencia pareció estar más vinculada a la alternancia de situaciones de hipoxia e hiperoxia.

\section{TIPOS DE TIEMPO, DENSIDAD DE OXÍGENO EN AIRE Y EFECTOS EN LOS INGRESOS HOS- PITALARIOS POR ENFERMEDADES DE RIESGO. ALGUNOS EJEMPLOS}

A fin de comprobar estas hipótesis de trabajo se han analizado los efectos de situaciones atmosféricas concretas y su relación con la presencia mayor o menor de oxígeno en el aire, en la salud de las personas residentes en la ciudad de Alicante.

Figura 1

LOCALIZACIÓN DEL ÁREA DE ESTUDIO. ESTACIONES METEOROLÓGICAS. DEPARTAMENTOS DE SALUD DE LA PROVINCIA DE ALICANTE

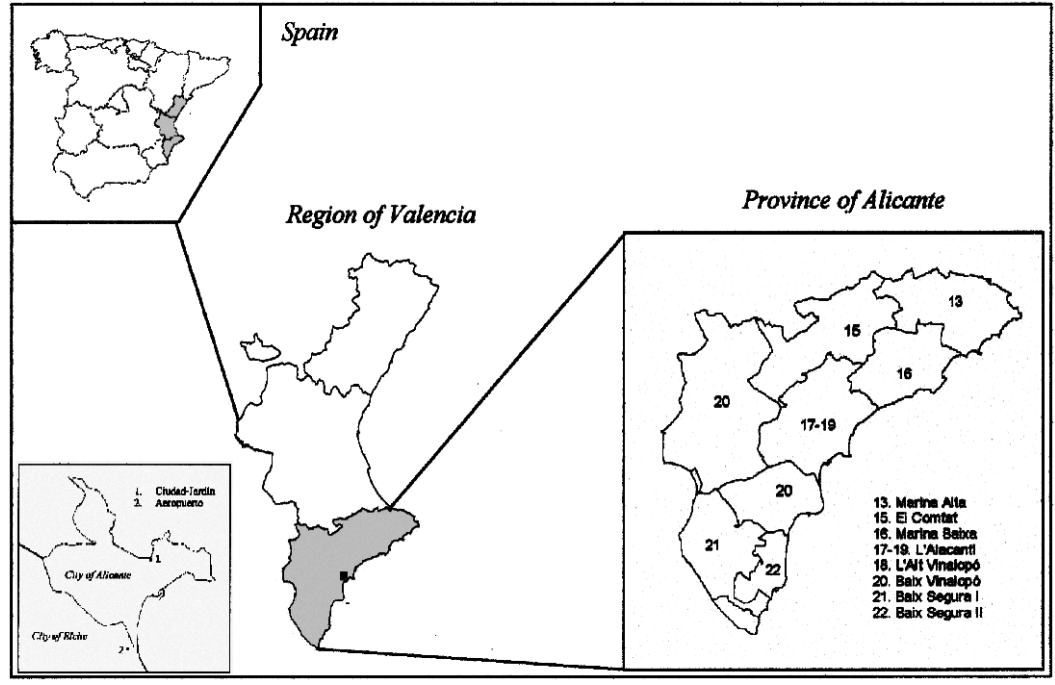

Fuente: Elaboración propia.

mas que se producen tienen relación con un conjunto de causas previas (factores de riesgo) que luego desencadenan el impacto, de ahí el concepto con significación más amplia de enfermedad. En nuestro caso, al no tratarse de un estudio médico, sino geográfico, donde nuestro objetivo es analizar las consecuencias derivadas de la variabilidad atmosférica que inciden en impactos en la salud, hemos optado por utilizar el término accidente en lugar de enfermedad, para referirnos a los ingresos hospitalarios de personas que han sufrido un impacto en el cerebro. Entendemos que el déficit o contraste en la densidad de oxígeno en aire, puede ser un desencadenante en momentos determinados de estas enfermedades y, por ello, aludimos a él como accidente que se da en un momento concreto y que puede contribuir, junto con las demás causas, a desencadenar el ingreso hospitalario. 


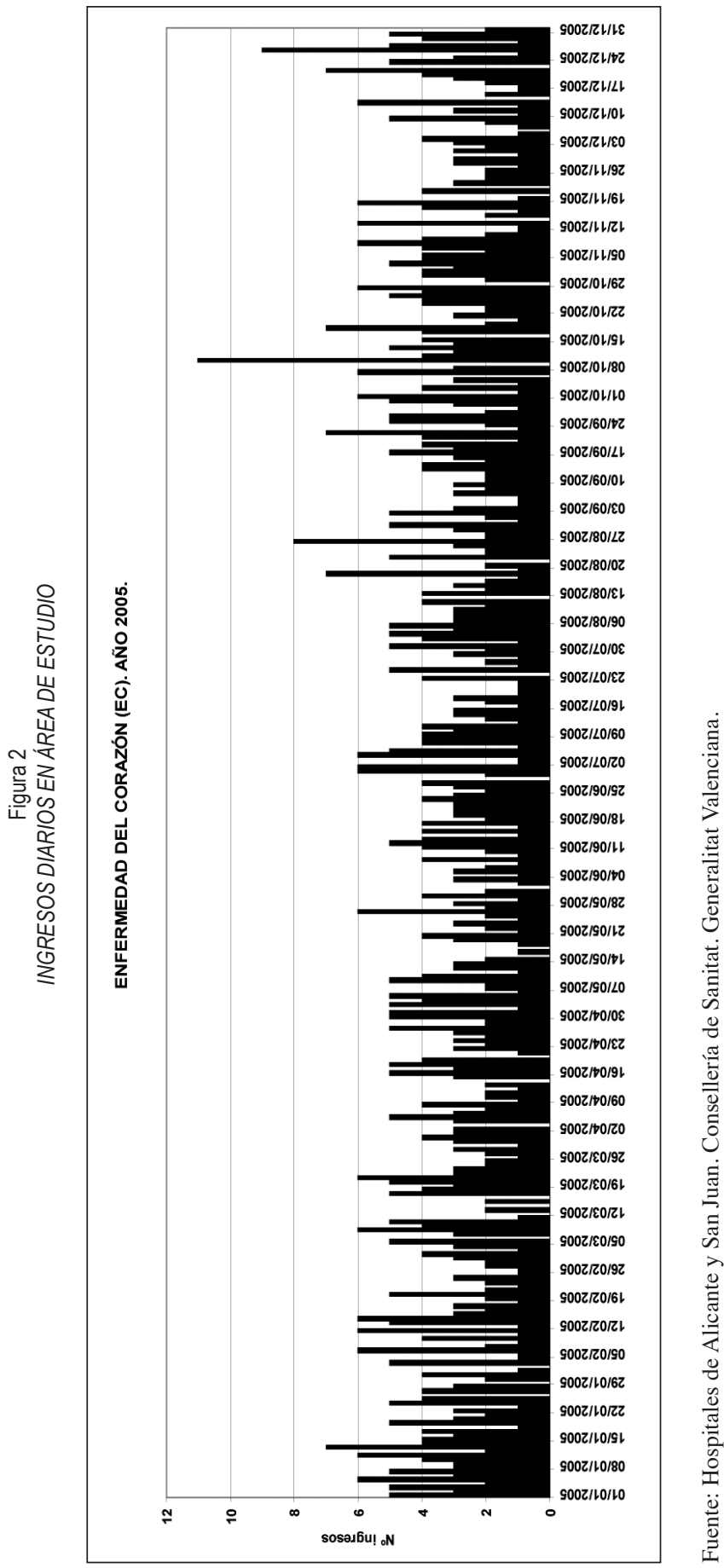


Se analiza la relación entre la variación del volumen de oxígeno en aire derivado del contraste atmosférico de las masas de aire y el aumento de los ingresos hospitalarios en personas incluidas en grupos de riesgo en los Departamentos de Salud ${ }^{13}$ de la ciudad de Alicante.

Se analizan dos situaciones, una de hipoxia y otra de contraste hipoxia-hiperoxia, para comprobar su influencia en el incremento de ingresos hospitalarios por afecciones cardiacas, pulmonares o vasculo cerebrales. Los datos meteorológicos analizados proceden de las dos estaciones oficiales que dispone la Agencia Estatal de Meteorología (AEMET) en el territorio de la ciudad de Alicante (Ciudad-Jardín y Aeropuerto).

\subsection{Análisis de episodio atmosférico con situación de hipoxia y aumento de los ingresos en enfermedades del corazón (EC)}

La primera situación se desarrolla entre los días 7 a 10 de octubre del año 2005. Ese episodio registró un importante número de ingresos hospitalarios relacionados con patologías cardíacas ${ }^{14}$. De modo especial la jornada del 10 de octubre registró un elevado número de ingresos, superando claramente el $150 \%$ de la media de los ingresos diarios en los dos hospitales analizados. Este valor es el umbral que se ha utilizado para caracterizar días normales y días extraordinarios, según el número de ingresos registrados (vid. Figura 2).

Desde comienzos de mes la instalación de un potente anticiclón centrado en el continente europeo favoreció la entrada de vientos fríos en el litoral mediterráneo español. En la jornada del 7 de octubre un embolsamiento de aire frío en altitud va desplazándose hacia el Mediterráneo occidental, mientras en superficie la configuración de vientos adquiere componente este (E). Ello provocó la entrada de vientos cargados de humedad favoreciendo el desarrollo de un ambiente atmosférico poco confortable. Esas dos circunstancias: presencia de los restos del embolsamiento de aire frío en altura y entrada de vientos húmedos del Mediterráneo en superficie, favorecieron una situación de contraste atmosférico con aumento de la temperatura y humedad relativa. En las jornadas siguientes (días 8, 9 y 10), la configuración isobárica propiciaría la entrada de vientos del sur-sureste en superficie y ello originó un nuevo aumento de la temperatura y de la humedad relativa (vid. Figura 3).

La entrada de aire sahariano, intensa desde la jornada del 9 de octubre, posibilitó un descenso significativo en el volumen de oxígeno en aire disponible (vid. Tabla 1). Ello coincidió con la jornada en que se registró el mayor número de ingresos hospitalarios por patologías cardiacas. Interesa destacar el efecto de acumulación en ese descenso del volumen de oxígeno en aire durante varias jornadas consecutivas ( 8 a 11 de octubre). A partir del 12 de octubre la humedad relativa desciende de forma considerable, la presión atmosférica aumentaría y ello provocó una mayor disponibilidad de oxígeno en aire.

La evolución de la topografía de $850 \mathrm{hPa}$ entre el 7 y el 10 de octubre permite apreciar, como se ha señalado, la llegada de aire sahariano y su permanencia en la atmósfera sobre el Sur y el Este de la península Ibérica a lo largo de estos días. Ello se manifestará con la seña-

13 Departamentos de Salud. División de la provincia de Alicante en áreas de atención hospitalaria de la Generalitat Valenciana según Orden de 12 de mayo de 2005, de la Consellería de Sanitat. En la ciudad de Alicante hay dos Departamentos: Alacant-Hospital General y Sant Joan d'Alacant.

14 Según la Clasificación Internacional de Enfermedades en su novena revisión (CIE-9: 410-41). 
Figura 3

EVOLUCIÓN DE LA SITUACIÓN SINÓPTICA EN LA TOPOGRAFÍA DE 500 HPA

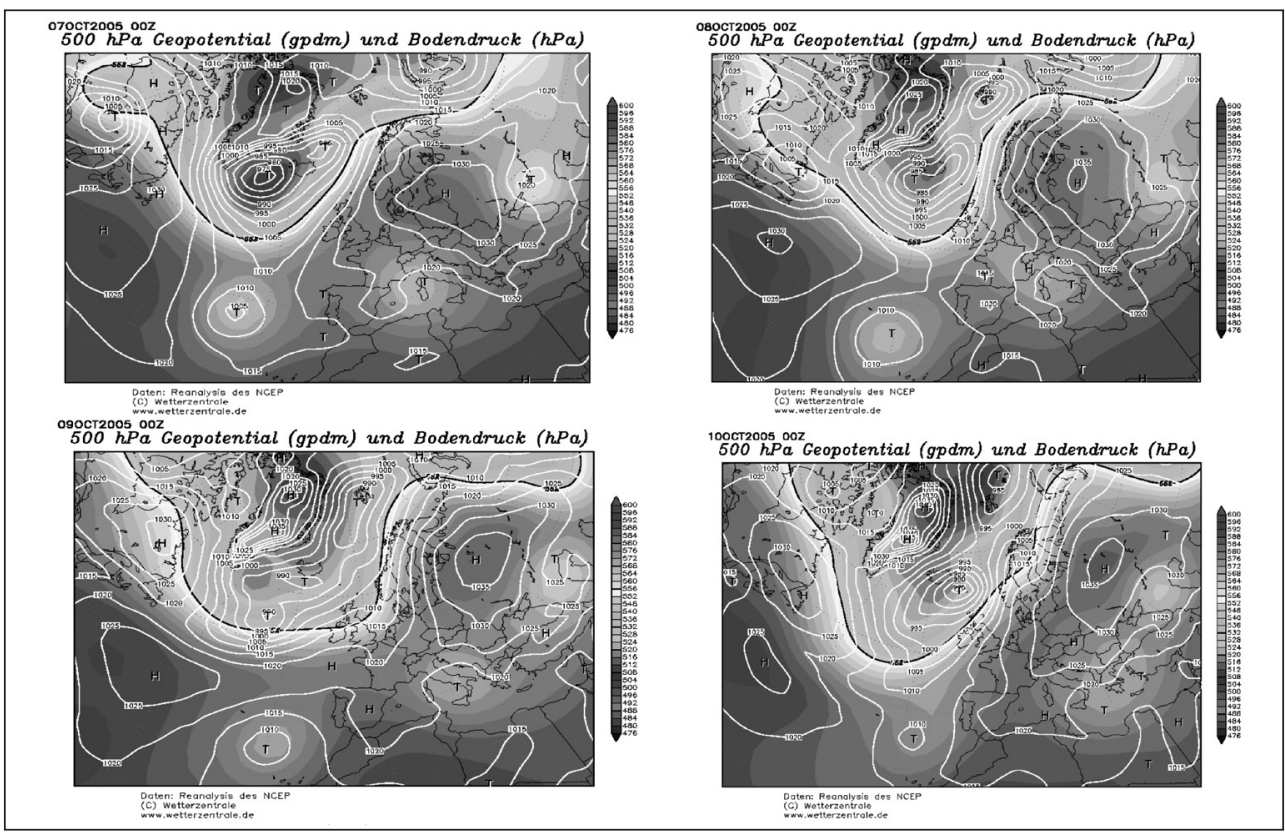

Fuente: www.wetterzentrale.de

Tabla 1

PARÁMETROS DE REFERENCIA PARA EL CASO DE ESTUDIO

\begin{tabular}{|c|c|c|c|c|c|c|}
\hline Día & $\mathrm{N}^{\mathrm{o}}$ casos & Tm & $\mathrm{Hm}$ & Pm & DOA & Var \\
\hline 05 -oct-2005 & 3 & 18,34 & 74,04 & 1018,54 & 277,11 & $-1,75$ \\
\hline 06-oct-2005 & 0 & 18,96 & 77,60 & 1017,42 & 275,82 & $-1,29$ \\
\hline 07 -oct-2005 & 6 & 19,70 & 73,98 & 1017,89 & 275,25 & $-0,57$ \\
\hline 08 -oct-2005 & 3 & 18,64 & 78,10 & 1019,03 & 276,63 & 1,38 \\
\hline 09-oct-2005 & 0 & 19,35 & 77,29 & 1020,70 & 276,25 & $-0,38$ \\
\hline 10 -oct-2005 & 11 & 20,54 & 80,54 & 1019,31 & 274,17 & $-2,09$ \\
\hline 11 -oct-2005 & 4 & 19,98 & 85,00 & 1015,53 & 273,55 & $-0,61$ \\
\hline 12 -oct-2005 & 3 & 21,11 & 85,75 & 1012,73 & 271,29 & $-2,27$ \\
\hline 13-oct-2005 & 5 & 19,69 & 57,29 & 1012,50 & 274,84 & 3,55 \\
\hline 14-oct-2005 & 3 & 17,11 & 65,96 & 1016,14 & 278,40 & 3,56 \\
\hline 15 -oct-2005 & 4 & 18,03 & 72,29 & 1018,50 & 277,58 & $-0,81$ \\
\hline 16-oct-2005 & 0 & 18,71 & 74,35 & 1018,50 & 276,62 & $-0,96$ \\
\hline 17-oct-2005 & 4 & 19,05 & 83,17 & 1014,87 & 274,67 & $-1,95$ \\
\hline 18 -oct-2005 & 7 & 19,93 & 86,71 & 1012,85 & 272,78 & $-1,90$ \\
\hline
\end{tabular}

( $\mathbf{N}^{\mathbf{o}}$ casos: Ingresos diarios de ésta patología; Tm: Temperatura media; Hm: Humedad media; Pm: Presión media; DOA: Densidad Oxígeno en Aire; Var: Variación del índice DOA interdiario).

Fuente: Hospitales de Alicante y San Juan de Alicante. Conselleria de Sanidad, Generalitat Valenciana. 
Figura 4

EVOLUCIÓN DE LA SITUACIÓN SINÓPTICA EN LA TOPOGRAFÍA DE 850 HPA

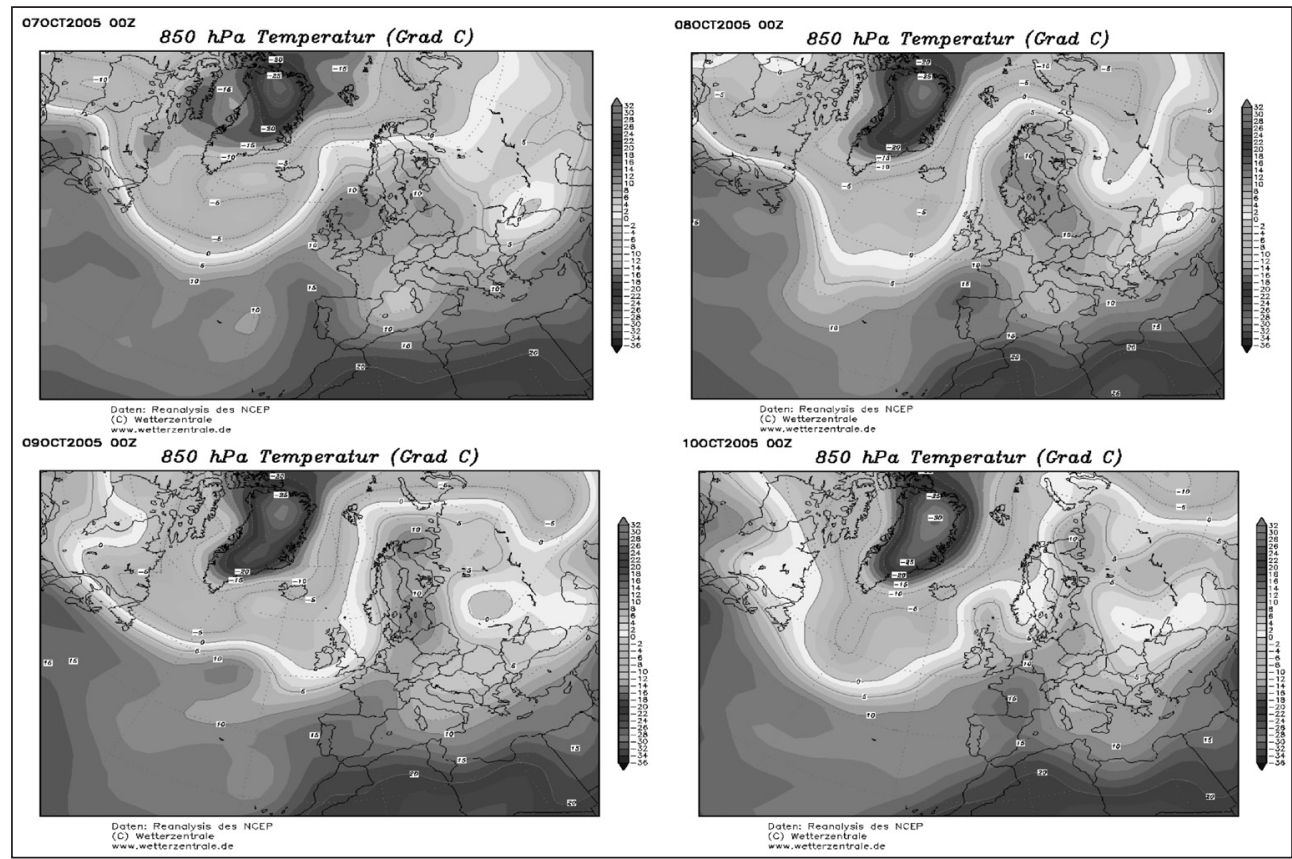

Fuente: www.wetterzentrale.de

lada reducción en el volumen de oxígeno en el aire y su relación con el número de ingresos hospitalarios por afección cardiaca ocurrido el día 10 de octubre (vid. Figura 4).

Debe indicarse que diferentes autores han señalado la dificultad de establecer un patrón claro de comportamiento en la relación entre volumen de oxígeno en aire e impacto en la salud de las personas que padecen enfermedades del corazón. Lo que si parece evidenciarse es que el desarrollo de contrastes bruscos en el volumen de oxígeno en aire puede ser un desencadenante en el desarrollo de afecciones en personas con patologías previas de corazón o en personas con algún factor de riesgo que puedan sufrir forzamiento en el funcionamiento de éste músculo por las variaciones ocurridas en el volumen de oxígeno en aire. En el gráfico adjunto (vid. Figura 5) se compara la modificación del volumen de oxígeno en aire y la evolución de los ingresos a lo largo de las jornadas del período de estudio. Independientemente de la condición biometeorológica que exista (en este caso, situación clara de hipoxia), es preciso señalar que un factor importante que puede contribuir al mayor impacto es el mantenimiento durante varias jornadas de unas condiciones anómalas en el volumen de oxígeno en aire. Ese contraste es el que se puede apuntar como factor determinante en la explicación del mayor número de ingresos por esta patología (enfermedades cardiacas) en el episodio que se analiza.

Por tanto, en este episodio se pueden apuntar como posibles causas para explicar el aumento significativo de los ingresos hospitalarios por enfermedades cardiacas en la ciudad 
Figura 5

EVOLUCIÓN DEL ÍNDICE DOA E INGRESOS HOSPITALARIOS EN LA CIUDAD DE ALICANTE

(5A 18 DE OCTUBRE DE 2005)

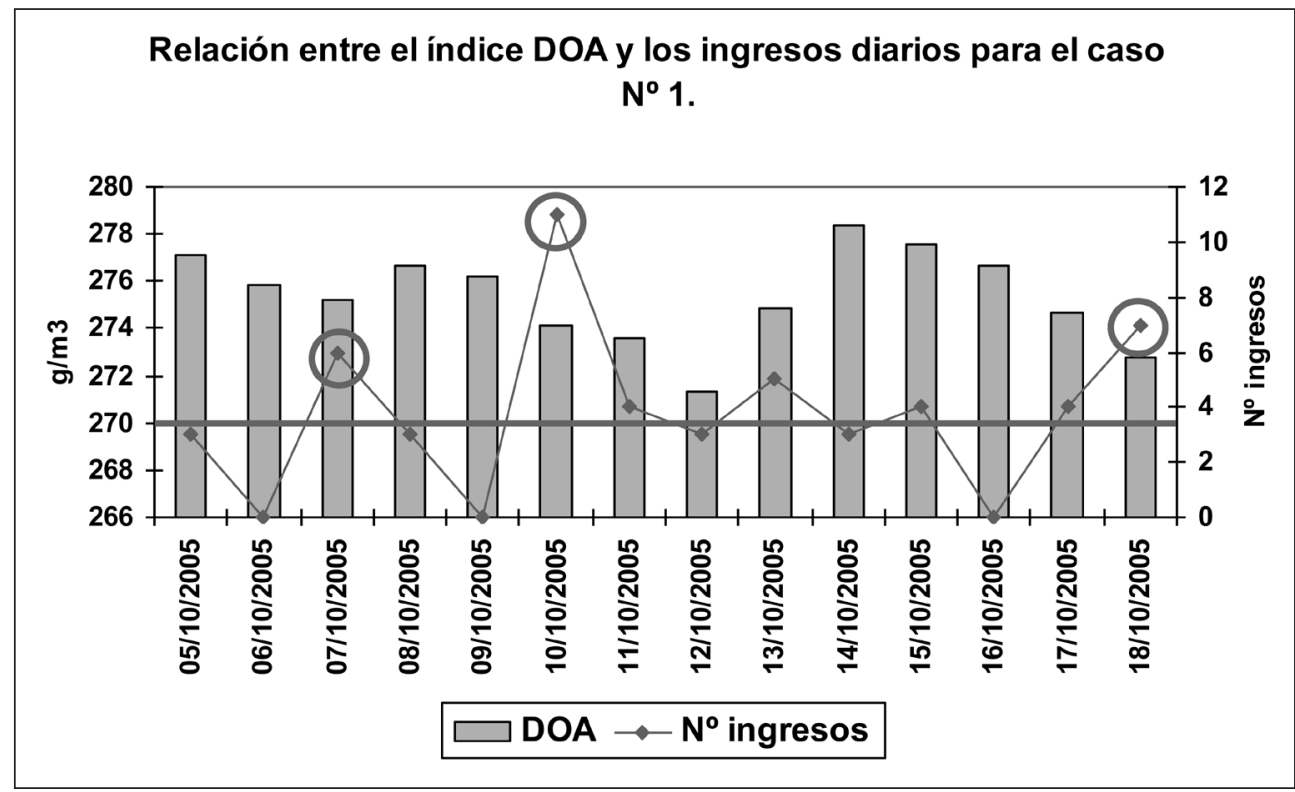

Fuente: Elaboración propia. Datos de los hospitales de referencia y del Laboratorio de Climatología del Instituto Interuniversitario de Geografía de la Universidad de Alicante.

de Alicante, el efecto combinado entre los contrastes de temperaturas y humedad relativa unido a la variación en la componente de los vientos predominantes en esas jornadas y la propia acumulación negativa en la disponibilidad de oxígeno en aire entre los días 7 y 10 de octubre.

\subsection{Descripción de la situación atmosférica con situación de contraste potencial de hipoxia y aumento de los ingresos relacionados con AVC entre finales de abril y principios de mayo de 2006}

Otro ejemplo significativo de la relación entre cantidad de oxígeno en aire y, en este caso, desarrollo de accidentes vasculo cerebrales (AVC) $)^{15}$ corresponde a la situación ocurrida entre finales de abril y comienzos de mayo de 2006, que generó un número importante de ingresos hospitalarios (en cuatro jornadas, se superó el umbral del 150\% de la media de los ingresos diarios en los dos hospitales de la ciudad de Alicante analizados) (vid. Figura 6).

15 AVC: Accidentes Vasculo Cerebrales. Según Clasificación Internacional de Enfermedades en su novena revisión CIE-9: 430-438. 


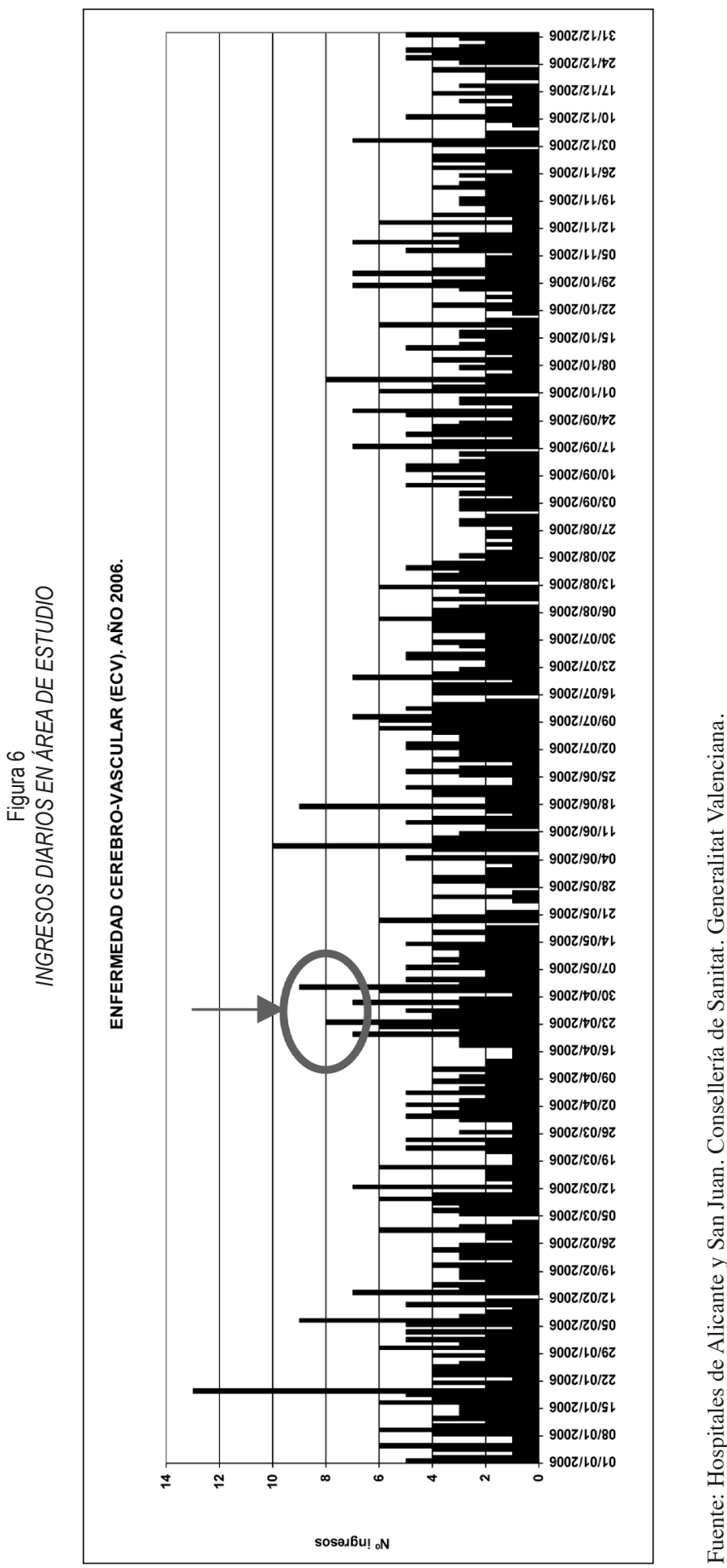


La situación atmosférica en los días que presentaron mayores ingresos hospitalarios se caracterizó, básicamente, por la presencia de vientos de componente SSE y temperaturas superiores a lo normal en esta época del año. Los mapas muestran la instalación de masas de aire contrastadas sobre la península Ibérica. Sobre el litoral mediterráneo y, especialmente en su sector sureste, destaca la entrada de aire del norte de África, lo que generó una situación de hipoxia en diversas jornadas del episodio analizado. A ello se unieron valores de humedad relativa altos, debido a la componente básica de vientos en superficie que favorecía el arrastre del aire sahariano sobre el mar Mediterráneo (vid. Figura 7).

Como en el caso anterior, estamos en una época del año equinoccial donde son constantes los cambios en el tiempo, motivados por la llegada de masas de aire de distinta naturaleza a la península Ibérica (polar y tropical). Estos contrastes produce situaciones con cambios bruscos en los parámetros atmosféricos y, derivado de ello, variaciones en la disponibilidad de oxígeno en aire. En estas condiciones, como se ha señalado, la necesidad de adaptación a las condiciones atmosféricas puede provocar que en individuos con factores de riesgo previo se produzca un impacto para la salud que puede derivar en el ingreso hospitalario (vid. Tabla 2).

Tabla 2

PARÁMETROS DE REFERENCIA PARA EL CASO DE ESTUDIO

\begin{tabular}{|c|c|c|c|c|c|c|}
\hline Día & $\mathbf{N}^{\mathbf{0}}$ casos & $\mathbf{T m}$ & $\mathbf{H m}$ & $\mathbf{P m}$ & DOA & Var \\
\hline $19 / 04 / 06$ & 3 & 15,55 & 73,69 & 1014,19 & 279,33 & 0,28 \\
\hline $20 / 04 / 06$ & $\mathbf{7}$ & $\mathbf{1 6 , 3 1}$ & $\mathbf{8 1 , 0 4}$ & $\mathbf{1 0 1 4 , 0 4}$ & $\mathbf{2 7 7 , 9 9}$ & $\mathbf{- 1 , 3 4}$ \\
\hline $21 / 04 / 06$ & 3 & 16,22 & 80,85 & 1012,28 & 277,62 & $-0,37$ \\
\hline $22 / 04 / 06$ & 6 & 16,24 & 86,50 & 1011,77 & 277,17 & $-0,45$ \\
\hline $23 / 04 / 06$ & $\mathbf{8}$ & $\mathbf{2 0 , 0 2}$ & $\mathbf{5 7 , 3 3}$ & $\mathbf{1 0 0 7 , 8 5}$ & $\mathbf{2 7 3 , 1 7}$ & $\mathbf{- 3 , 9 9}$ \\
\hline $24 / 04 / 06$ & 4 & 20,13 & 54,40 & 1007,01 & 273,01 & $-0,17$ \\
\hline $25 / 04 / 06$ & 4 & 20,80 & 41,17 & 1011,98 & 274,50 & 1,49 \\
\hline $26 / 04 / 06$ & 5 & 20,16 & 49,13 & 1016,67 & 275,97 & 1,47 \\
\hline $27 / 04 / 06$ & 3 & 18,39 & 73,10 & 1014,74 & 276,05 & 0,08 \\
\hline $28 / 04 / 06$ & $\mathbf{7}$ & $\mathbf{1 6 , 7 3}$ & $\mathbf{8 5 , 8 5}$ & $\mathbf{1 0 1 2 , 5 6}$ & $\mathbf{2 7 6 , 8 0}$ & $\mathbf{0 , 7 6}$ \\
\hline $29 / 04 / 06$ & 3 & 16,70 & 82,42 & 1011,53 & 276,74 & $-0,07$ \\
\hline $30 / 04 / 06$ & 1 & 16,71 & 84,38 & 1010,16 & 276,24 & $-0,50$ \\
\hline $01 / 05 / 06$ & 6 & 18,14 & 77,98 & 1010,38 & 274,87 & $-1,37$ \\
\hline $02 / 05 / 06$ & $\mathbf{9}$ & $\mathbf{1 8 , 6 3}$ & $\mathbf{6 7 , 9 2}$ & $\mathbf{1 0 0 9 , 7 5}$ & $\mathbf{2 7 4 , 6 9}$ & $\mathbf{- 0 , 1 8}$ \\
\hline $03 / 05 / 06$ & 3 & 19,98 & 70,77 & 1006,34 & 271,93 & $-2,76$ \\
\hline $04 / 05 / 06$ & 5 & 18,15 & 63,38 & 1010,94 & 275,87 & 3,94 \\
\hline
\end{tabular}

( $\mathbf{N}^{\mathbf{o}}$ casos: Ingresos diarios de ésta patología; Tm: Temperatura media; Hm: Humedad media; Pm: Presión media; DOA: Densidad Oxígeno en Aire; Var: Variación del índice DOA interdiario).

Fuente: Hospitales de Alicante y San Juan de Alicante. Conselleria de Sanidad, Generalitat Valenciana. 
Figura 7

EVOLUCIÓN DE LAATMÓSFERAA 850 HPA ENTRE LOS DÍA 19 DE ABRIL Y 3 DE MAYO DE 2006
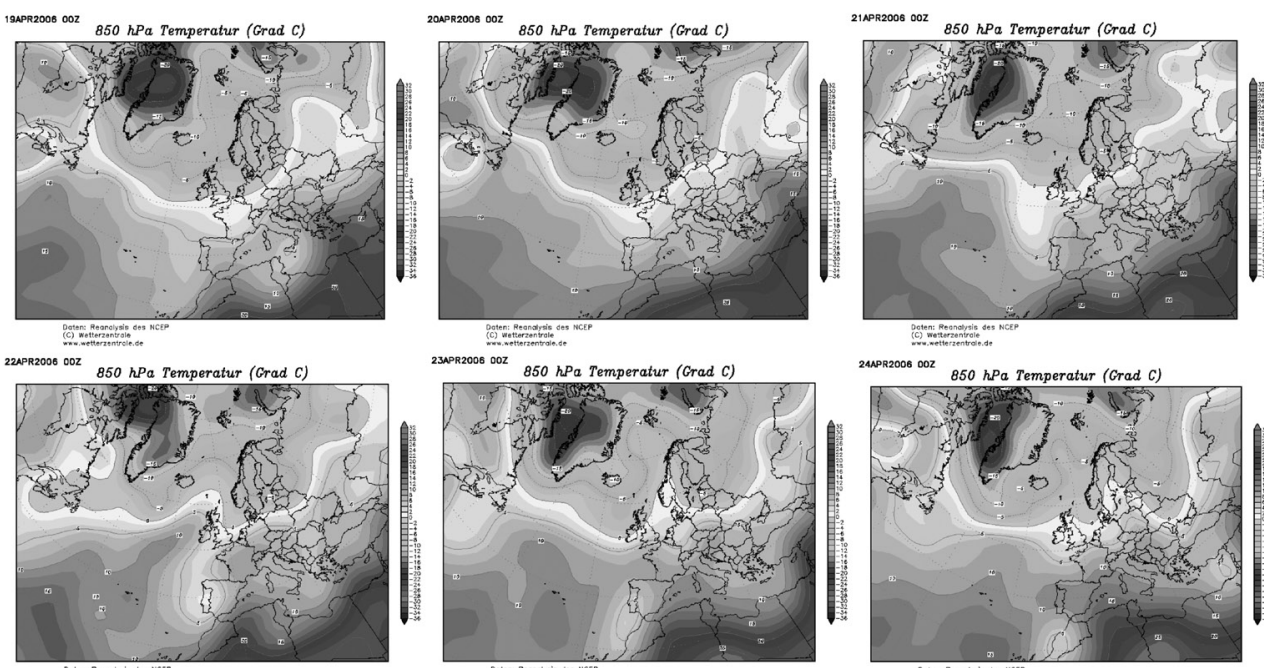

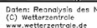
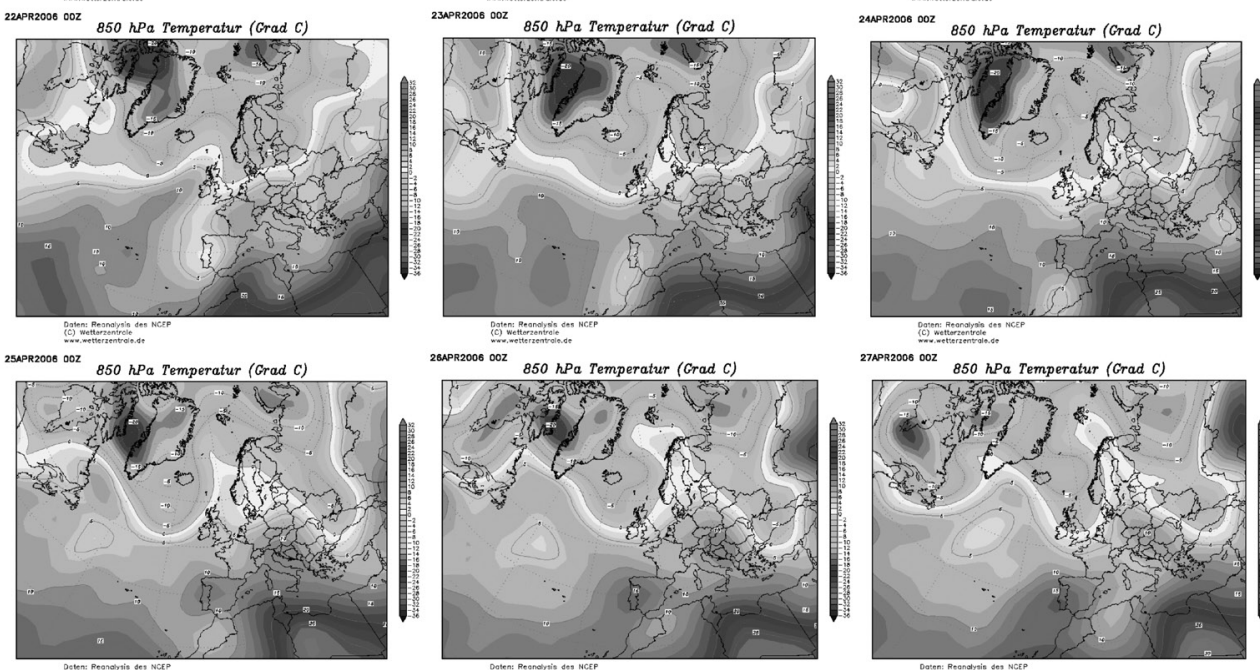

28APR2006 002 , 850 if
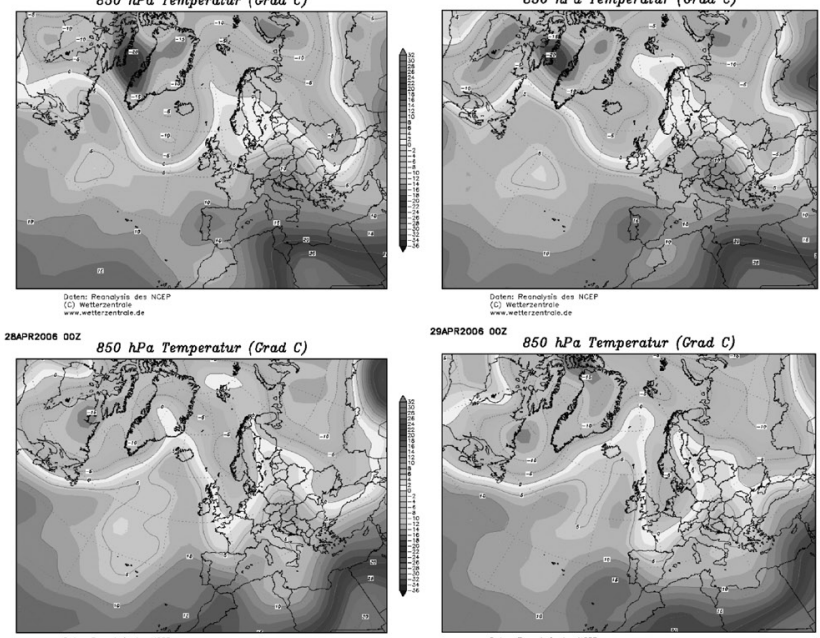

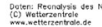

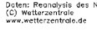
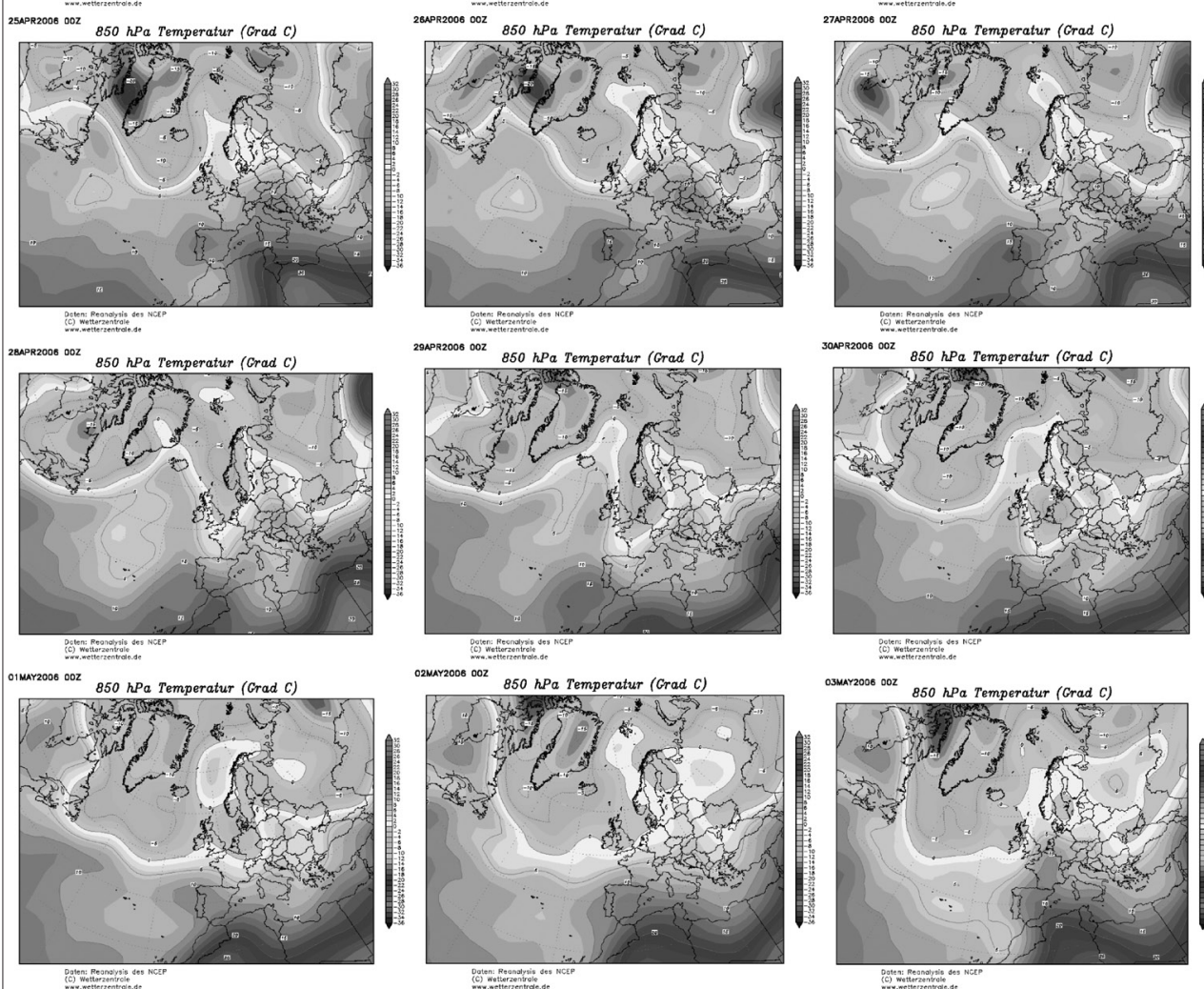

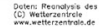

03 Marzoov $00 \mathrm{~B} \quad 850 \mathrm{hPa}$ Temperatur (Grad C)

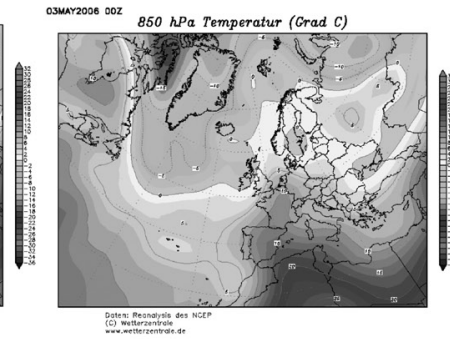

Fuente: http://www.wetterzentrale.de/topkarten/fsreaeur.html 
En los días que componen el episodio analizado se registraron ingresos superiores a tres en los dos hospitales de referencia, destacando dos jornadas -23 de abril y 2 de mayo - con número de ingresos superior a siete (vid. Tabla 2). Estas dos jornadas coinciden con una variación negativa acumulada de oxígeno en aire causada por la presencia de la masa de aire tropical continental, con varias secuencias de advección (20-21 de abril; 23-25 abril; 28 de abril y 1 a 3 de mayo). Entre estas advecciones de aire tropical se desarrollan jornadas con niveles de oxígeno normal o ligeramente superior a lo normal, debido a la instalación de aire frío en el espacio sinóptico peninsular. De esta forma, el contraste en la presencia de oxígeno en el aire puede predisponer a determinadas personas sensibles a sufrir un impacto y pudo ser el origen del aumento significativo de ingresos hospitalarios por impacto vásculo cerebral (vid. Figura 8).

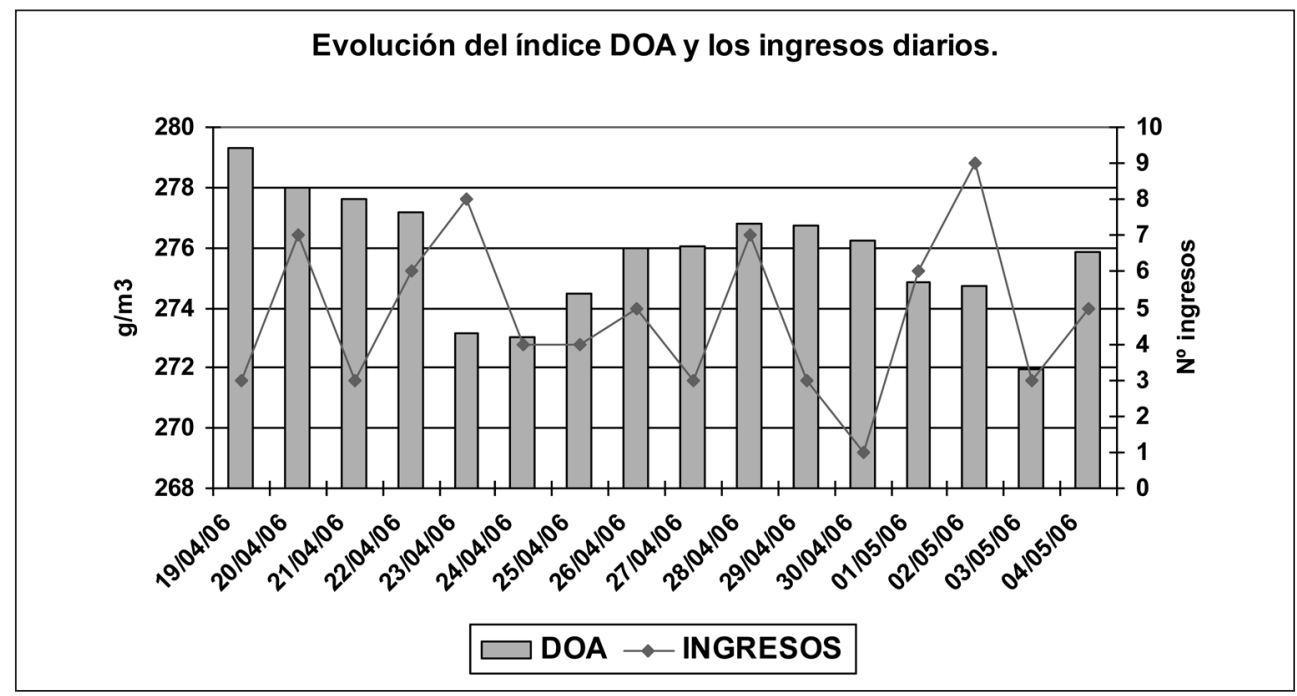

Fuente: Elaboración propia: Datos hospitales de Alicante y San Juan y Laboratorio de Climatología del Instituto Internuniversitario de Geografía (Universidad de Alicante).

\section{ESTABLECIMIENTO DE SISTEMAS DE ALERTA TEMPRANA A LAS POBLACIONES EN RELACIÓN CON LA PRESENCIA DE OXÍGENO EN EL AIRE}

Una de las cuestiones que, en la actualidad, interesa dentro de la investigación entre presencia de oxígeno en el aire y posibles afecciones a la salud humana es el establecimiento de sistemas de alerta temprana a las poblaciones. Estos sistemas de alerta temprana, que funcionan ya en algunas regiones del mundo, son de fácil aplicación por las administraciones y en ellos se establecen los protocolos necesarios para minimizar el impacto de situaciones atmosféricas con falta o superávit de oxígeno. 
La disponibilidad de oxígeno en aire para desarrollar las funciones vitales se ve condicionada por la evolución de las condiciones atmosféricas. Si éstas presentan cambios bruscos, como suele ser normal en latitudes medias en los meses de transición al verano y al invierno, es decir, primavera y otoño, la presencia de un sistema de alerta temprana puede ayudar a minimizar los riesgos. Estos sistemas establecerían diferentes grados de aviso previo, posibilitando que los profesionales de la salud, el establecimiento de protocolos de actuación (p.e. la utilización de oxígeno medicinal) al objeto de intentar reducir las posibles crisis de los pacientes con patologías previas. Puede servir de referencia el proceso de adaptación que deben llevar a cabo los alpinistas que ascienden relieves de gran altitud y que, si no llevan a cabo un programa de adaptación a la altitud, pueden padecer patologías pulmonares o vasculo cerebrales severas debido a la falta de oxígeno en alta montaña (a $8.000 \mathrm{~m}$. de altitud sólo se dispone de un porcentaje de oxígeno en el aire correspondiente al 37\% del existente a nivel del mar); de ahí que sea necesario la utilización de botellas de oxígeno para evitar el riesgo, muchas veces mortal, del conocido como «mal de altura».

Existen desde hace años sistemas de aviso ante episodios atmosféricos de rango extraordinario, donde se incluyen los generados por temperaturas máxima o mínimas extremas. Así, en Estados Unidos, la Agencia Federal de Emergencias y la NOAA disponen también de un sistema de avisos para pronosticar las olas de calor y frío, con protocolos de actuación para los ciudadanos. En España tanto la Agencia Estatal de Meteorología (AEMET) como los organismos meteorológicos de diferentes Comunidades Autónomas disponen de un sistema de avisos que elabora mapas diarios de alerta por temperaturas extremas. Con ellos se avisa de la probabilidad de desarrollo de situaciones peligrosas y se establecen protocolos de trabajo a desarrollar por protección civil. Este mecanismo de avisos forma parte del programa europeo «Meteoalarm» que integra a treinta países europeos en un sistema en red de información sobre eventos atmosféricos extremos.

A ellos se han unido sistemas de alerta biometeorológica donde se incorporar indicadores de confort climático en relación con las condiciones atmosféricas existentes. Es el caso del programa «PronBiomet», protocolo de alerta temprana desarrollado en Cuba, que parte de la elaboración de mapas de pronóstico biometeorológicos a gran escala, con indicación de configuraciones de hipoxia e hiperoxia. En estos mapas se incluye, asimismo, la distribución de los campos de presión en superficie. De manera que la lectura e interpretación de estos mapas de pronóstico permite establecer un catálogo de situaciones sinópticas (tipos de tiempo) más o menos proclives a desarrollar situaciones de hipóxia e hiperóxia, aspecto previo para el establecimiento posterior de alertas a la población. En Alemania, por su parte, se ha desarrollado un servicio de pronósticos biometeorológicos (MedizinID ${ }^{\mathrm{TM}}$-System) que incluye valores de un nuevo índice universal de ambiente térmico ${ }^{16}$ para las distintas regiones del país.

De manera que a los sistemas de alerta meteorológica tradicionales, que avisan de la posible ocurrencia de episodios atmosféricos de rango extremo, se han sumado nuevos sistemas de alerta orientados al aviso de consecuencias para la salud humana de situaciones

16 Universal Termal Climate Index (UTCI). Se trata de un índice complejo que incorpora diferentes parámetros atmosféricos (temperatura efectiva, radiación, humedad y viento). El índice trata de homogeneizar, a nivel global, los resultados de índices anteriores. Puede consultarse en www.utci.org 
atmosféricas en relación con la presencia mayor o menor de oxígeno en el aire o basados en otros índices biometeorológicos complejos cuyo objetivo es establecer estadios o grados de aviso a la población en relación con el ambiente atmosférico circundante para ese momento y para las jornadas venideras.

A partir de los mapas de pronóstico biometeorológicos elaborados dentro del proyecto «PronBiomet» puede servir como ejemplo de estos mecanismos de aviso a las poblaciones el episodio ocurrido en la última quincena de marzo de 2009, que ocasionó un número de ingresos de accidentes vasculo cerebrales superiores a la media diaria en los centros hospitalarios de la ciudad de Alicante. La situación atmosférica de las dos semanas finales de este mes de marzo se caracterizó por el desarrollo de una secuencia meteorológica con cambios constantes de temperie. La situación se inicia con unas jornadas iniciales de tiempo anticiclónico con entrada de vientos del norte sobre el litoral mediterráneo español y ambiente frío que, tiene como consecuencia, el aumento del volumen de oxígeno en aire creando una situación de hiperoxia, como se puede apreciar en los mapas biometeorológicos (vid. Figura 9).

Figura 9

SITUACIÓN ATMOSFÉRICA Y BIOMETEOROLÓGICA DEL 17 DE MARZO DE 2009

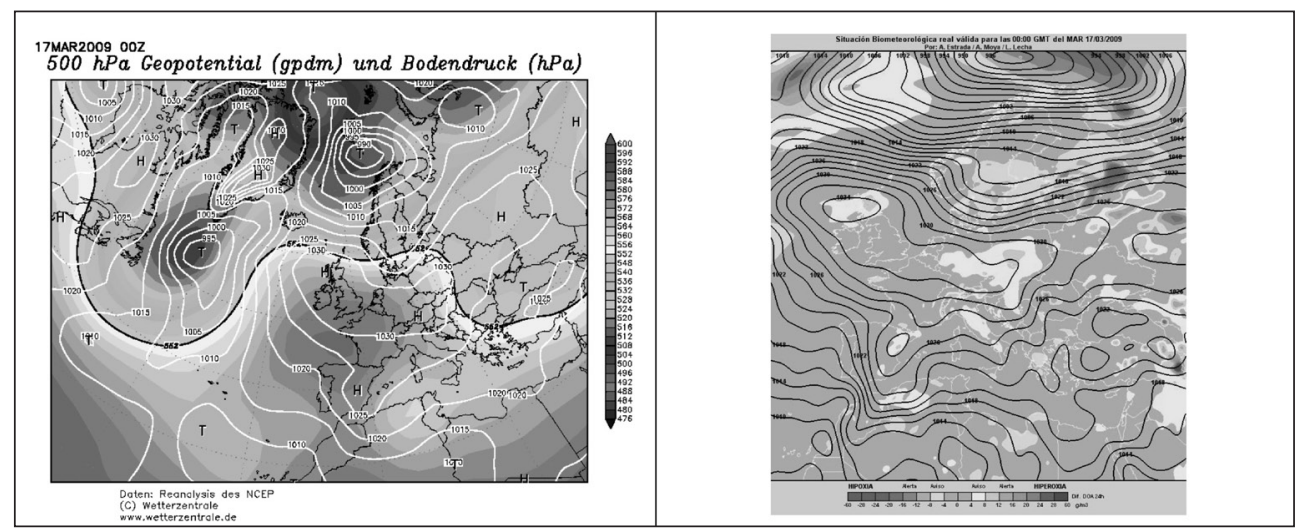

Fuente: www.wetterzentrale.de y Lecha Estela, L. et al. Sistema de Alerta Temprana Global PromBiomet.

A partir del 20 de marzo la llegada de una masa de aire polar continental, procedente del este de Europa, hacia el Mediterráneo occidental y la península Ibérica, ocasionará una modificación intensa en las condiciones atmosféricas, con instalación de bajas presiones e inestabilización general del tiempo, lo que conllevaría una reducción del volumen de oxígeno en aire generando una situación de hipoxia en el litoral mediterráneo español (vid. Figura 10). Se trata de condiciones atmosféricas propicias para el establecimiento de un «aviso» biometeorológico por una hipoxia moderada, puesto que en esta situación se puede disparar el aumento diario de casos de hipertensión arterial, cefaleas y accidentes vásculo cerebrales de tipo isquémico. Estas condiciones se prolongarían hasta el día 22 de marzo.

A partir del 23 de marzo se produce una advección de masa de aire cálida (tropical continental) sobre la península Ibérica y, que impondría jornadas de tiempo estable y subida de temperaturas hasta finales de mes. Las presiones experimentan un ligero incremento el día 23 
Figura 10

SITUACIÓN ATMOSFÉRICA Y BIOMETEOROLÓGICA DEL 20 DE MARZO DE 2009

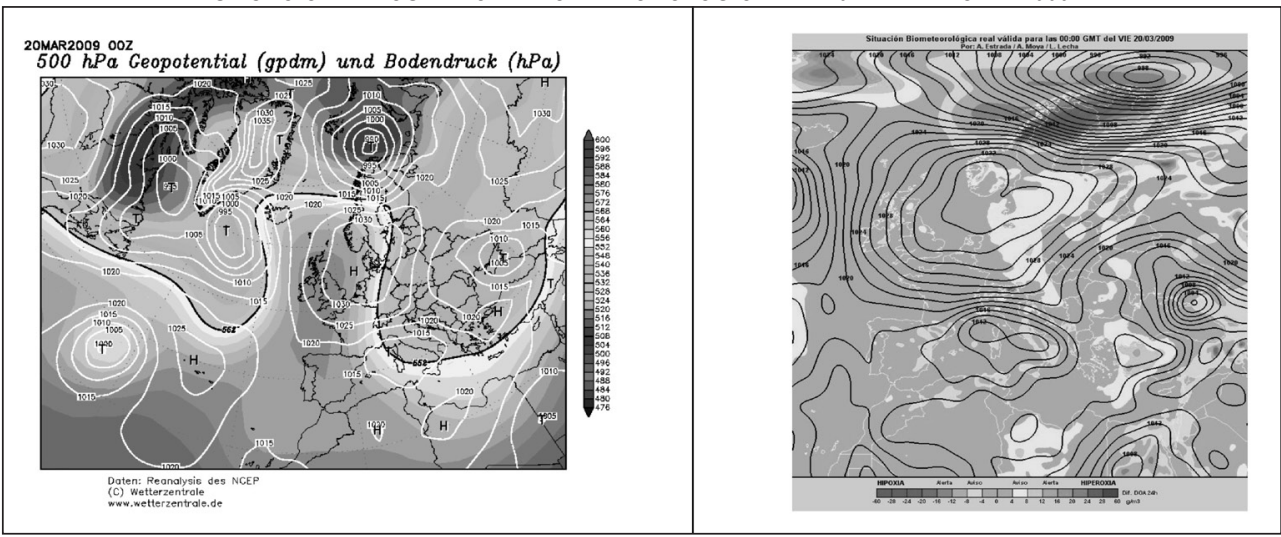

Fuente: www.wetterzentrale.de y Lecha Estela, L. et al. Sistema de Alerta Temprana Global PromBiomet.

para descender a partir del día 24, en relación con la presencia de un talweg barométrico en el norte de África que termina abrazando el sur de las tierras ibéricas. En estas condiciones se mantendrán las condiciones de hipoxia que venían registrándose desde el 20 de marzo y que se agudizaran los días 24 y 25, especialmente sobre la mitad meridional de la península Ibérica (Vid. Figura 11).

Figura 11

SITUACIÓN ATMOSFÉRICA Y BIOMETEOROLÓGICA DEL 25 DE MARZO DE 2009

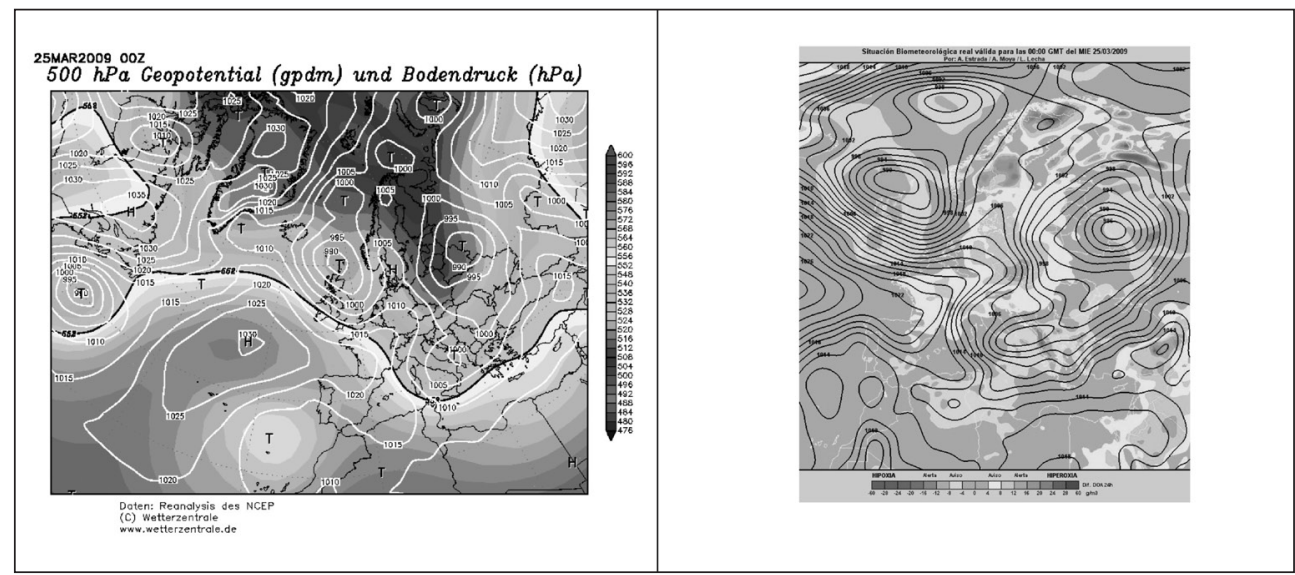

Fuente: www.wetterzentrale.de y Lecha Estela, L. et al. Sistema de Alerta Temprana Global PromBiomet.

Este contraste hiperoxia-hipoxia en pocas jornadas tuvo un impacto significativo en la ciudad de Alicante, a tenor de los datos de ingresos hospitalarios registrados por accidente vásculo cerebral, puesto que el día 24 se registran cuatro ingresos y alcanzan los nueve ingre- 
sos el día 26. Se trata de una situación prototípica que recomendaría el establecimiento de un sistema de aviso biometeorológico a la población. Y ello porque si se conoce con antelación una situación de contraste en la presencia de oxígeno en aire, - en éste caso de hipoxia- , merced a los pronósticos biometeorológicos manejados en un sistema de alerta temprana, la administración encargada de la salud pública podría recomendar la aplicación de terapias preventivas como forma de compensación de la caída de oxígeno a causa de movimientos de masas de aire y de los cambios en los campos isobáricos en superficie.

\section{CAMBIO CLIMÁTICO, PRESENCIA DE OXÍGENO EN EL AIRE Y AUMENTO FUTURO DE ENFERMEDADES CARDIOVASCULARES Y PULMONARES}

Los modelos de cambio climático del IPCC (2007) y la adaptación regional que ha llevado a cabo en España la Agencia Estatal de Meteorología (AEMET), indican la probable modificación de las condiciones climáticas en la región mediterránea española que supondría, con incremento de temperaturas medias y máximas, descenso de precipitaciones y un aumento de la irregularidad atmosférica, esto es, la probabilidad de que aumente la frecuencia de desarrollo de fenómenos extremos en este territorio. Para la región de la Comunidad Valenciana, las proyecciones climáticas establecidas sobre los escenarios A1 y, concretamente, el escenario $\mathrm{A}_{1 \mathrm{~B}^{17}}$, se recogen en la figura adjunta (vid. Figura 12).

Figura 12

PROYECCIÓN DE TEMPERATURAS MÁXIMAS, MÍNIMAS Y PRECIPITACIÓN EN LA COMUNIDAD VALENCIANA (ESPAÑA) PARA EL SIGLOXXI

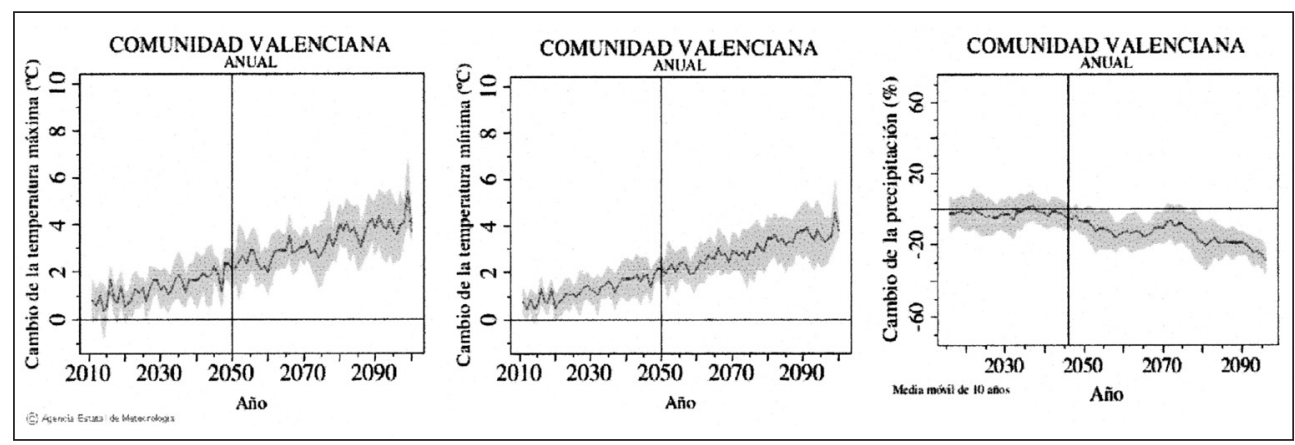

Fuente: Proyecto ENSEMBLES. Agencia Estatal de Meteorología (AEMET).

17 La línea evolutiva y familia de escenarios A1 describe un mundo futuro con un rápido crecimiento económico, una población mundial que alcanza su valor máximo hacia mediados del siglo y disminuye posteriormente, y una rápida introducción de tecnologías nuevas y más eficientes. Sus características distintivas más importantes son la convergencia entre regiones, la creación de capacidad y el aumento de las interacciones culturales y sociales, acompañadas de una notable reducción de las diferencias regionales en cuanto a ingresos por habitante. La familia de escenarios A1 se desarrolla en tres grupos que describen direcciones alternativas del cambio tecnológico en el sistema de energía. Los tres grupos A1 se diferencian en su orientación tecnológica: utilización intensiva de combustibles de origen fósil (A1FI), utilización de fuentes de energía no de origen fósil (A1T), o utilización equilibrada de todo tipo de fuentes (A1B). El término equilibrada de éste último escenario indica que no se dependerá excesivamente de un tipo de fuente de energía, en el supuesto de que todas las fuentes de suministro de energía y todas las tecnologías de uso final experimenten mejoras similares. 
Como líneas generales, se prevé para el sector central del litoral mediterráneo español un aumento de la temperatura máxima en los próximos cuarenta años de $2^{\circ} \mathrm{C}$, que subiría hasta $4^{\circ} \mathrm{C}$ a finales del presente siglo, de no llevar a cabo las medidas necesarias de reducción de gases de efecto invernadero. Las temperaturas mínimas muestran una tendencia similar. Por su parte, la tendencia de las precipitaciones manifiesta una reducción significativa que podría llegar al $20 \%$ en la segunda mitad de siglo. A ello se uniría, como se ha señalado, la probabilidad de que los tipos de tiempo tengan un comportamiento menos regular, debido a las alteraciones en los procesos de reajuste energético y al movimiento más dinámico de las masas de aire. Ello puede tener unas implicaciones muy importantes en la salud de los seres humanos que pueden verse afectados por secuencias de temperatura extrema más intensas.

$\mathrm{Al}$ incremento de la peligrosidad climática previsto para las próximas décadas, se une el aumento de la vulnerabilidad social en este territorio, puesto que el litoral mediterráneo español es uno de los focos de atracción de población procedente de diferentes áreas de España y de países del centro y norte de Europa. Además del incremento de población estacional que ocurre en los meses de verano, relacionado con la actividad turística, desde los años noventa del siglo XX se asiste a la llegada de residentes europeos que han encontrado en el sureste

Figura 13

PORCENTAJE DE EXTRANJEROS PROCEDENTES DE EUROPA Y POBLACIÓN MAYOR DE 65 AÑOS POR PROVINCIAS EN ESPAÑA

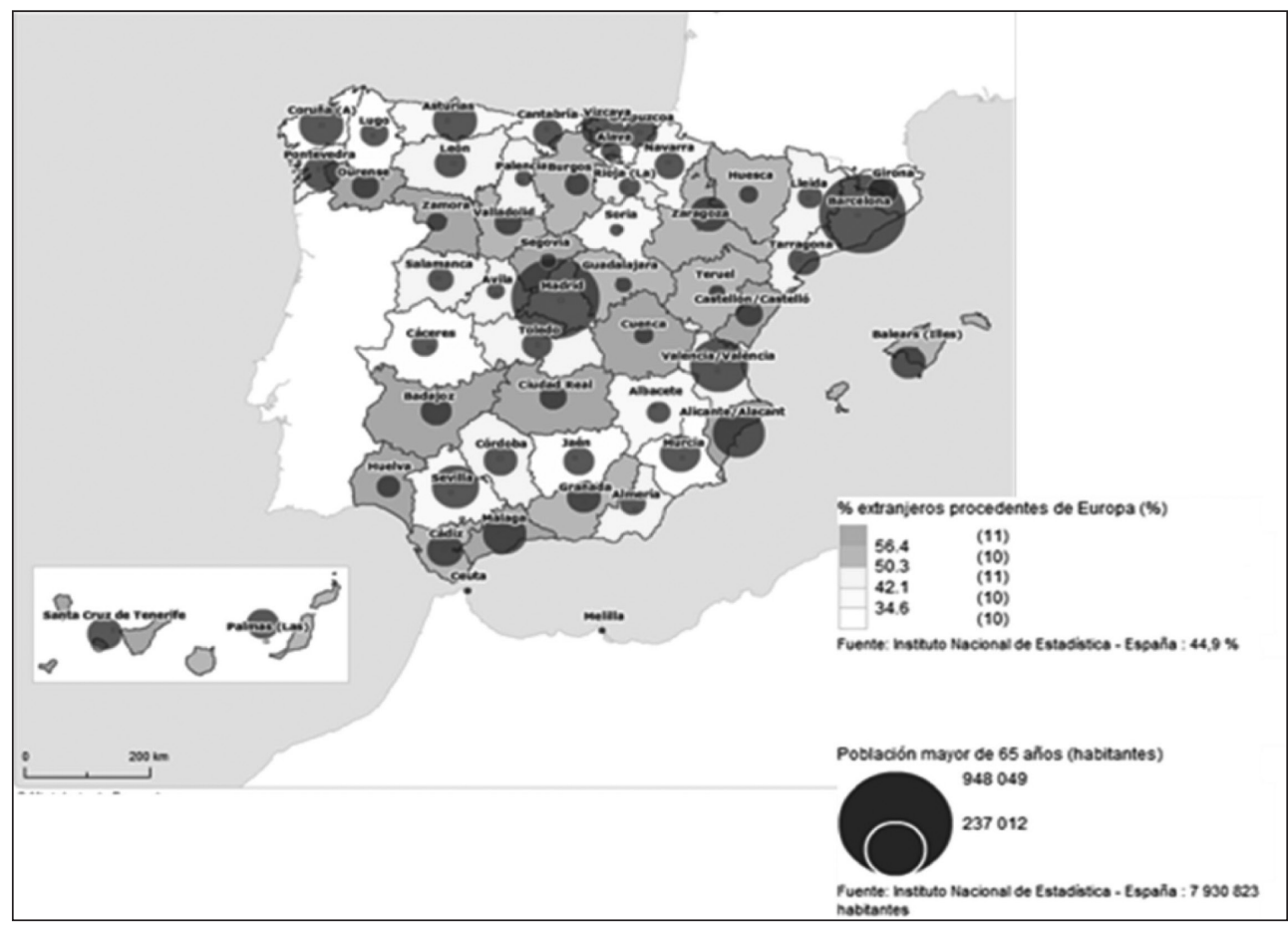

Fuente: Atlas Digital de las Áreas Urbanas. Ministerio de Fomento. 
peninsular el territorio propicio para desarrollar su período final de vida. El grupo de población mayoritario en este proceso ha sido, por tanto, el de las personas mayores de 65 años. Ello, unido a una estructura de la población española, cada vez más envejecida y con menor reemplazo generacional, ha provocado un importante aumento en el grupo poblacional de personas mayores. Se trata de un proceso no exclusivo del territorio alicantino que ha afectado a buena parte del litoral mediterráneo español y al archipiélago canario, y en el que se ha cimentado un porcentaje importante de la actividad inmobiliario-residencial que ha sido el motor del desarrollo económico de estos territorios hasta la reciente crisis económica (vid. Figura 13).

En relación con los temas de salud, la presencia de este importante grupo poblacional de personas mayores ha favorecido, en las dos últimas décadas, el incremento de la vulnerabilidad social al tratarse de personas con salud generalmente más delicada y muy sensibles a los cambios bruscos del tiempo atmosférico. Si a ello se añade la señalada previsión de aumento significativo de los fenómenos meteorológicos extremos, señalados en los modelos de cambio climático (IPCC, 2007), el litoral mediterráneo español y el archipiélago canario se pueden convertir en áreas de alerta para la salud humana y por tanto de atención prioritaria en el diseño de sistemas de prevención sanitaria.

En efecto, una de las características climáticas en latitudes medias es la variabilidad climática no sólo estacional, sino dentro de ella, la variabilidad que acontece dentro del mismo día. Diferentes estudios han analizado los impactos de estas variaciones en la salud de las personas. Modificaciones en la temperatura o la humedad, provocan cambios en la temperatura corporal, la respiración, el ritmo cardiaco y la circulación sanguínea. El intercambio de calor está íntimamente ligado al proceso metabólico, que a su vez, se encuentra ligado al sistema nervioso (Fernández, 1995). El organismo, de forma natural, entra en un proceso de adaptación a esos cambios y ello sucede en todas las regiones del planeta. Estos cambios, que pueden llegar a ser muy severos, no sólo dificultan las actividades humanas, sino que pueden poner en peligro la propia vida (Dolfus, 1975).

Las consecuencias del cambio climático en la salud de las personas se presenta como un importante reto a gestionar. La tendencia al crecimiento de las ciudades durante los próximos cincuenta años, aumentará la vulnerabilidad de la salud las personas, debido a la confluencia de diferentes factores: aumento de las temperaturas debido a la variación climática, aumento paulatino del fenómeno de isla de calor urbana en nuestras ciudades, aumento de la contaminación en las mismas, aumento de la esperanza de vida de las personas, aumento de las personas incluidas en los llamados grupos de riesgo y mayor número de personas con patologías crónicas que se pueden ver afectadas por eventos meteorológicos extremos. Estos factores deben ser analizados y se precisa afrontarlos desde una perspectiva multidisciplinar, puesto que afectan a expertos de formación diversa.

En este sentido, se han desarrollado diversos estudios que vinculan temperaturas extremas con la mortalidad. De sus resultados se puede decir que hay una coincidencia entre episodios de temperatura extrema e incremento de la mortalidad. Para el caso de los episodios de frío intenso destacan los trabajos de Kunst et al., 1993; Mitchell, 2001 y Analitis et al., 2008. En condiciones de frío duradero el organismo humano intenta impedir un descenso del calor orgánico que pueda comprometer a las funciones vitales. Con la presencia de las bajas temperaturas, se produce una reacción orgánica de vasoconstricción periférica, modificando 
el flujo sanguíneo para evitar las pérdidas de calor a través de la piel. Ello, puede provocar, con temperaturas muy bajas, riesgo de fallo cardíaco debido al incremento de la presión sanguínea como consecuencia de la vasoconstricción. Esa reacción fisiológica, produce una reducción del volumen de sangre y, lógicamente, una disminución del oxígeno encargado de mantener las funciones vitales (Thomas, 2004). Healy (2003) apunta diferencias en cuanto a las tasas de mortalidad relacionadas con las bajas temperaturas entre países del norte y del sur, siendo éstos últimos los que mayores tasas presentan por la menor costumbre del cuerpo humano a experimentar estas condiciones térmicas extremas.

Los efectos de las bajas temperaturas sobre la salud, al contrario de lo que sucede con los episodios de olas de calor, pueden manifestarse a lo largo de las dos o incluso tres semanas siguientes de suceder el episodio (Hales et al., 2003). En el litoral mediterráneo español, el estudio llevado a cabo por Analitis et alt. (2008) estima que un descenso de $1{ }^{\circ} \mathrm{C}$, aumentaría la mortalidad natural en los grupos de más edad y en los grupos de más de 65 años por problemas respiratorios. Por el contrario, aumentaría en todos los grupos de edad la mortalidad relacionada con las enfermedades cardiovasculares. En cuanto a la mortalidad por accidentes vasculo cerebrales, el descenso de la temperatura afectaría a los grupos mayores de 75 años en Barcelona, en cambio, en la ciudad de Valencia, esta última patología afectaría en mayor medida a los menores de 65 años.

En relación con el desarrollo de infartos de miocardio por efecto de temperaturas muy bajas a las personas con esta patología, Wolf et alt. (2009) han señalado que su incidencia suele ser destacada en invierno, pero el impacto puede ser asimismo destacado si se da en meses estivales tras producirse un descenso puntual, no habitual, de las temperaturas. A tenor de lo comentado, y siguiendo las tesis del IPCC respecto al aumento de las temperaturas en diferentes regiones del planeta, se puede señalar un aspecto positivo, y es que, muchas patologías relacionadas con el frío, pueden llegar a reducirse de manera considerable por efecto del aumento de las temperaturas en un futuro cercano en las regiones afectadas por ese aumento de las temperaturas.

Por su parte, resulta más compleja la relación entre episodios atmosféricos de elevadas temperaturas y defunciones. En estos casos las defunciones se suelen producir prácticamente al momento (golpes de calor) o, en los dos días siguientes al episodio de ola de calor (Dessai, 2002). Los umbrales térmicos a partir de los cuales se experimenta un incremento acusado de la mortalidad dependen de las características geográficas y climáticas de cada país o región. Así, en Canadá, se considera ola de calor el episodio donde en tres o más días consecutivos se superan las máximas de $32{ }^{\circ} \mathrm{C}$, mientras que en Dallas (EEUU) la ola de calor se define como la presencia de $37,8^{\circ} \mathrm{C}$ consecutivos durante un mínimo de tres días (Hales, Edwars y Kovats, 2003). En España los umbrales de temperatura establecidos a escala provincial en el sistema de avisos de fenómenos meteorológicos adversos se mueven entre $26,2{ }^{\circ} \mathrm{C}$ en La Coruña y $41,2{ }^{\circ} \mathrm{C}$ en Córdoba (Díaz et al., 2005).

Diferentes autores han analizado los efectos de las olas de calor acontecidas en los últimos años. Destacan los trabajos sobre efectos en Chicago (Estados Unidos) de la ola de calor de junio de 1995 (Semenza et al., 1996); en el mismo verano Londres registró un aumento de la mortalidad de $16 \%$ (Rooney et al., 1998). Y especialmente, numerosos son los trabajos sobre efectos del tórrido verano de 2003 en Europa occidental, donde algunos estudios establecieron una cifra de víctimas mortales de 30.000 personas (Kovats y Jendritzki, 2006). 
Sólo en Francia se calcularon 15.000 muertes (un $70 \%$ de esta cifra correspondiente al grupo de edad de mayores de 75 años). En la ciudad de París, durante el período del 1 al 15 de agosto, se registraron 5.000 muertes atribuibles a los efectos del calor extremos (vid. Figura 14). En este mismo episodio, la ciudad de Barcelona registró un aumento de la mortalidad del 16,6 \% más (Martínez-Navarro et al., 2004).

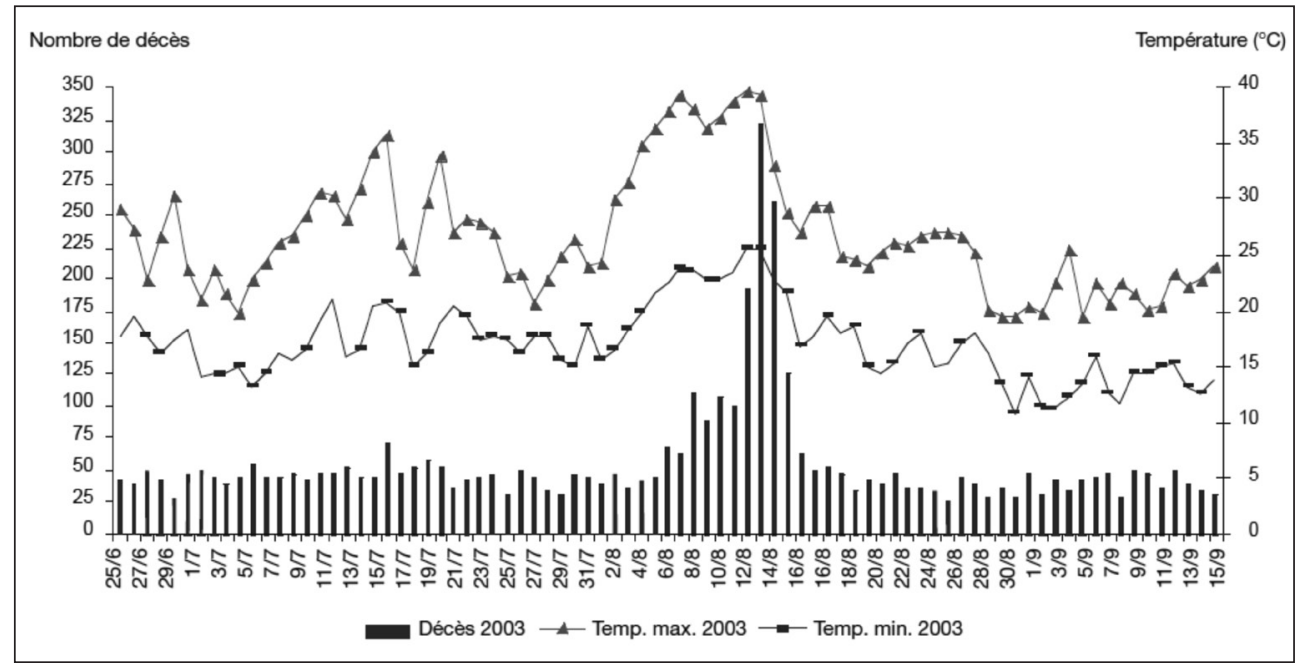

Fuente: Institute de Veille Sanitaire.

\section{CONCLUSIONES}

Las proyecciones climáticas para las próximas décadas señalan un aumento de las temperaturas (medias y máximas), así como el incremento de la variabilidad atmosférica y de su carácter extremo en el litoral mediterráneo español. A ello se une un incremento de la vulnerabilidad por el aumento de grupos de riesgo de enfermedades cardiovasculares y pulmonares, motivado por la tendencia al envejecimiento natural de la población en nuestro país, así como a la llegada de residentes europeos, especialmente en la fachada este peninsular.

En el análisis sobre la relación entre presencia de oxígeno en el aire y desarrollo potencial de enfermedades cardiacas, pulmonares y vásculo cerebrales realizado para la ciudad de Alicante cabe señalar que le hecho de que una situación atmosférica favorezca una mayor o menor densidad de oxígeno no es causa directa del desarrollo de estas dolencias, pero si puede actuar como elemento favorecedor de su desarrollo en grupos de riesgo (personas mayores de 65 años) y personas con afección previa de dichas enfermedades.

Destaca el hecho de que las enfermedades cardiacas muestran una estacionalidad marcada, siendo los tres primeros y los tres últimos meses del año los que recogen el mayor 
número de ingresos, coincidiendo con los meses más fríos. Ahora bien, se evidencia también un número importante de ingresos por esta afección durante los meses estivales, con resultado de muerte de los pacientes. Similares conclusiones cabe señalar para los accidentes vasculo cerebrales que muestran también estacionalidad de los ingresos en el invierno, aunque presentan impactos en época estival, afectando más a mujeres que a hombres.

La evolución del contenido de oxígeno en la atmósfera (DOA), a la largo del año en la ciudad de Alicante, adquiere una disposición en forma de «V» con máximos en los primeros y últimos meses del año, mientras que los meses centrales, sobre todo estivales, presenta los mínimos anuales. Los valores extremos para la ciudad de Alicante ${ }^{18}$ oscilan entre los $295 \mathrm{~g} /$ $\mathrm{m}^{3}$ y $260 \mathrm{~g} / \mathrm{m}^{3}$.

El descenso acumulado del volumen de oxígeno en aire en varias jornadas consecutivas pone a prueba el organismo humano, pudiendo producir impactos en la salud. Con ésta situación atmosférica se notaron incrementos en AVC y en EC, aunque en ésta última patología parecen más difusos. Para los AVC, se observa que la presencia de contraste en el volumen de oxígeno en aire dos días antes y dos días después puede ser determinante, ya que el organismo puede responder a cambios tipo «descenso-ascenso-descenso» de la DOA en aire en un período corto de tiempo.

Un porcentaje elevado de los ingresos (70\%), coinciden con situaciones en las que se produce un descenso acumulado negativo del índice DOA. Si bien, juega un papel importante el contraste de masas de aire en corto intervalo de tiempo, esto es, el juego de situaciones de hiperoxia e hipoxia en pocos días.

De ello se deduce la importancia de los sistemas de alerta temprana que estimen la variación del volumen de oxígeno en aire disponible por exceso o por defecto. Ello pueden contribuir a minimizar los impactos de estos cambios ambientales a las personas incluidas en grupos de riesgo (personas susceptibles de padecer enfermedad cardiovascular) e igualmente, a personas con patologías relacionadas con el aparato respiratorio.

A tenor de las señaladas previsiones de los modelos de cambio climático para las próximas décadas, en la región mediterránea, resultaría necesaria la implantación de sistemas de alerta temprana que incluyan protocolos de actuación sanitaria que minimicen situaciones de riesgo potencial para la salud de todas las personas sensibles a los cambios de oxígeno en el aire.

\section{AGRADECIMIENTOS}

Queremos expresar nuestro agradecimiento a la Agencia Estatal de Meteorología de España (AEMET), el haber puesto a disposición los datos que han sido utilizados en este trabajo. Igualmente, al Dr. D. Luis Lecha Estrela y su equipo de Centro de Estudios Ambientales de Villa Clara (Cuba) por poner a nuestra disposición el sistema de alerta temprana de la variación de la densidad de oxígeno en aire (índice DOA), con su cartografía biometeorológica. Nos gustaría agradecer, de la misma manera, al Departamento de Epidemiología de la Dirección General de Salud Pública en la Consellería de Sanitat de la Generalitat Valenciana, la amabilidad para facilitarnos los datos de salud empleados en este trabajo y, especialmente,

18 Martín Estévez, David (2010): Op. cit. nota 10. 
a los responsables de los dos Departamentos de salud de Alicante (Alacant-Hospital General y Sant Joan D'Alacant). Junto a ellos, también nuestro agradecimiento a Dña. Inmaculada Melchor Alós.

\section{BIBLIOGRAFÍA}

ALIAGA P., A.; ESTRADA C., M.; PÉREZ P., N.; SEGURA B., A.; SERRA F., J. (2006): Mens sana in corpore sano. La salud en la época romana. ACV Ediciones, Barcelona, España.

ANALITIS A.K, et al. (2008): «Effects of Cold Weather on Mortality: Results From 15 European Cities Within the PHEWE Project». American Journal of Epidemiology, 168: 1397-1408.

DESSAI, S. (2002): «Heat stress and mortality in Lisbon Part I. model construction and validation», International Journal of Biometeorology, 47: 6-12.

DÍAZ, J.; LINARES, C.; GARCÍA-HERRERA, R. (2005): «Impacto de las temperaturas extremas en la salud pública: futuras actuaciones». Revista Española de Salud Pública. 79: $145-147$.

DOLLFUS O. (1975): El espacio geográfico. Oikos- Tau, Barcelona.

FERNÁNDEZ DE ARRÓYABE H., P.; LECHA E., L. (2008): «Validación en el norte de España de dos sistemas de alerta sanitarios basados en la idea del contraste meteorológico extremo», En Cambio climático regional y sus impactos.VI Congreso Internacional AEC. Serie A, ponencia V. Tarragona 2008.

FERNÁNDEZ GARCÍA, F. (1995): Manual de climatología aplicada. Clima, medioambiente y planificación. Ed. Síntesis. Madrid, 288 p.

GLACKEN, C. J (1996): Huellas en la playa de Rodas. Naturaleza y cultura en el pensamiento occidental desde la Antigüedad hasta finales del siglo XVIII. Ediciones del Serbal, Barcelona, 729 pp.

HALES, S.; EDWARDS, S.J.; KOVATS, R.S. (2003): «Impacts of health of climate extrems», in McMICHAEL, A.J., et al. (eds.) Climate Change and Human Health. World Health Organization, Ginebra, p. 79-102.

HEALY, J.D. (2003) : «Excess winter mortality in Europa: a cross country analysis identifying key risk factors», Journal of Epidemiology and Community Health, 57: 784-789.

KOVATS, R.S.; JENDRITZKY, G. (2006): «Heat-waves and human health», in. Menne, B.; Ebi, K.L., (eds.) Climate change and adaptation strategies for human health. Darmstadt, Springer, p. 63-97.

KUNST A., E.; LOOMAN C., W.N.; MACKENBACH J. P. (1993): «Outdoor air temperature and mortality in the Netherlands: a time-series analysis». American Journal of Epidemiology, 137: 331-341.

LECHA ESTELA, L. (1998): «Biometeorological classification of daily weather types for the humid tropics», International Journal of Biometeorology, Volume 42, Number 2, Pages 77-83.

LECHA ESTELA, L., (2007): «Pronósticos para la mitigación de los impactos del tiempo sobre la salud humana», Simposio Cambio Climático y Salud. VI Congreso Nacional de Epidemiología e Higiene. La Habana, Cuba. 
LECHA ESTELA, L., E.; CIÓMINA E.C., G.; ESTRADA A. (2007): «Los pronósticos biometeorológicos: una vía para reducir las crisis de salud en la población cubana», en Memorias del IV Congreso Cubano de Meteorología, La Habana, Cuba.

MARTÍNEZ, N. F.; SIMÓN, S. F.; LÓPEZ, A. G. (2004): «Valoración del impacto de la ola de calor del verano de 2003 sobre la mortalidad», Gaceta Sanitaria, 18, pp. 250-258.

MITCHELL, R. (2001) «Short days-shorter lives: studying winter mortality to gat solutions», International Journal of Epidemiology, 30 pp. 1116-1118.

OVCHAROVA, V.F. (1981): «Calculation of oxygen content in the air on the basis of meteorological parameters (pressure, temperature and humidity) for the prediction of the effects of hypoxic conditions», Rev. Climatotherapy problems, Physiotherapy and Physical Culture, 2, Moscú, pp. 29-34, (in Russian).

RASO NADAL, J.M (2010): «Salut. El Canvi Climàtic a Catalunya», 2 n Informe del Grup d'Experts en Canvi Climàtic de Catalunya, Generalitat de Catalunya, Barcelona, pp. 913-959.

ROONEY, C. et al.,(1998): «Excess mortality in England and Wales, and in Greater London, during the 1995 heatwave», Journal of Epidemiology and Community Health, 52, pp. 482-486.

SEMENZA, J.C., et al., (1996): «Heat-related deaths during the July 1995 heat wave in Chicago», The New England Journal of Medicine, 335, pp. 84-90.

THOMAS, P. (2004): Under the Weather. Fusion, Londres.

WOLF, K. et al., (2009): «Air temperature and the occurrence of myocardial infarction in Augsburg, Germany», Circulation, 9, pp. 735-742. 